\title{
Physicochemical models: source-tailored or generic?
}

\author{
Beatrice M. Kulterer ${ }^{\circledR},{ }^{1 \star}$ Maria N. Drozdovskaya ${ }^{\circledR},{ }^{1}$ Audrey Coutens ${ }^{\circledR},{ }^{2}$ Sébastien Manigand ${ }^{3}$ \\ and Gwendoline Stéphan ${ }^{4}$ \\ ${ }^{1}$ Center for Space and Habitability, University of Bern, Gesellschaftsstrasse 6, CH-3012 Bern, Switzerland \\ ${ }^{2}$ Laboratoire d'astrophysique de Bordeaux, Univ. Bordeaux, CNRS, B18N, allée Geoffroy Saint-Hilaire, F-33615 Pessac, France

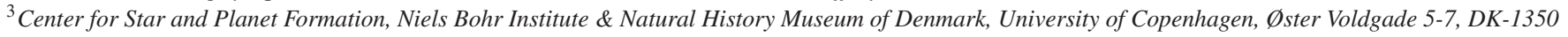 \\ Copenhagen K., Denmark \\ ${ }^{4}$ Department of Chemistry, University of Virginia, Charlottesville, VA 22904, USA
}

Accepted 2020 August 10. Received 2020 August 10; in original form 2020 May 22

\begin{abstract}
Physicochemical models can be powerful tools to trace the chemical evolution of a protostellar system and allow to constrain its physical conditions at formation. The aim of this work is to assess whether source-tailored modelling is needed to explain the observed molecular abundances around young, low-mass protostars or if, and to what extent, generic models can improve our understanding of the chemistry in the earliest stages of star formation. The physical conditions and the abundances of simple, most abundant molecules based on three models are compared. After establishing the discrepancies between the calculated chemical output, the calculations are redone with the same chemical model for all three sets of physical input parameters. With the differences arising from the chemical models eliminated, the output is compared based on the influence of the physical model. Results suggest that the impact of the chemical model is small compared to the influence of the physical conditions, with considered time-scales having the most drastic effect. Source-tailored models may be simpler by design; however, likely do not sufficiently constrain the physical and chemical parameters within the global picture of star-forming regions. Generic models with more comprehensive physics may not provide the optimal match to observations of a particular protostellar system, but allow a source to be studied in perspective of other star-forming regions.
\end{abstract}

Key words: astrochemistry-protoplanetary discs - stars: protostars - ISM: abundances.

\section{INTRODUCTION}

Cold, dense cores may be starless, prestellar, or protostellar in nature. Initially, they are all characterized by a spatial extent in the range of $\sim 0.1 \mathrm{pc}$, temperatures of $\sim 10 \mathrm{~K}$, and typical densities of a few $10^{4} \mathrm{~cm}^{-3}$ (Benson \& Myers 1989; Bergin \& Tafalla 2007). With time these slowly rotating cores with a typical rotation rate ( $\Omega$ ) of $\sim 1 \mathrm{~km} \mathrm{~s}^{-1} \mathrm{pc}^{-1}$ (Shu, Adams \& Lizano 1987; Goodman et al. 1993) can concentrate mass. If their central density increases above $10^{5} \mathrm{~cm}^{-3}$, they are deemed to be prestellar and display signs of kinematic and chemical evolution (e.g. Crapsi et al. 2005; Keto \& Caselli 2008). Once they become unstable and collapse in an insideout manner, a central protostar accompanied by a disc is formed (Shu et al. 1987). Both are enshrouded by an infalling envelope of dust and gas, which accretes on to the disc and the protostar. If the formation and destruction rates of molecules are quantified, they can function as diagnostic tools of the evolving physical structures of these systems, because the rates of the chemical processes depend on the physical conditions and time. Comparing observations to predicted model abundances gives insights into the history of such systems.

Since the first detection of an interstellar molecule in the 1930s (e.g. Swings \& Rosenfeld 1937), more than 200 have been detected in the interstellar medium (ISM; McGuire 2018). While the majority of

^E-mail: beatrice.kulterer@csh.unibe.ch the detected species consists of two or three atoms, complex organic molecules (COMs) have been detected in prestellar cores and around protostars of all masses (e.g. Agúndez et al. 2019; Gieser et al. 2019). Following the definition given by Herbst \& van Dishoeck (2009), a $\mathrm{COM}$ consists of at least six atoms and contains the element carbon. The simplest in structure is methanol $\left(\mathrm{CH}_{3} \mathrm{OH}\right)$, which is frequently detected in the ISM (e.g. Leurini et al. 2016; Guzmán et al. 2018; Chacón-Tanarro et al. 2019). Its formation occurs on dust grain surfaces at dust temperatures of $\sim 12 \mathrm{~K}$ by subsequent hydrogenation of $\mathrm{CO}$, which has been quantified by theoretical studies and in the laboratory (e.g. Tielens \& Hagen 1982; Fuchs et al. 2009). Öberg et al. (2009b) showed in laboratory experiments that radicals, produced by the photodissociation of UV-irradiated (ultraviolet) methanol ices, proceed to form species with a higher degree of complexity, e.g. glycolaldehyde $\left(\mathrm{CH}_{2} \mathrm{OHCHO}\right)$. Moreover, laboratory studies produced species as complex as glycerol $\left(\mathrm{HOCH}_{2} \mathrm{CH}(\mathrm{OH}) \mathrm{CH}_{2} \mathrm{OH}\right)$ starting from the hydrogenation of $\mathrm{CO}$ (Fedoseev et al. 2017). Thus, a variety of the observed complex species is believed to form on icy interstellar grains (Watanabe \& Kouchi 2008).

Astrochemical models provide a powerful tool to link observed molecules in interstellar space to theoretical and experimental findings. Physical parameters (e.g. gas density, gas and dust temperatures, and UV flux) are derived from observational data, which are then analysed following radiative transfer theory. The derived physical parameters can then be used to tailor a model specifically towards the observed source or serve as input for a model that aims to 
provide a more generic picture of a typical source of its type. Models can target a single structure of a system, a certain time during the system's evolution, or give its full evolutionary history. For instance, dedicated models have been developed for protoplanetary discs (e.g. Walsh et al. 2014; Schwarz et al. 2018; Wakelam et al. 2019). Others investigated the chemical evolution of pre- and protostellar systems (e.g. Taquet, Charnley \& Sipilä 2014; Vasyunin et al. 2017) or connected astrochemical modelling to planet formation models to investigate the composition of planetary embryos (e.g. Cridland, Eistrup \& van Dishoeck 2019). Input for the chemical networks used in models is gained from laboratory experiments, where several processes are considered over a range of physical conditions for a selection of molecules, including processes in the gas phase and in ices, as well as theoretical computations. Physicochemical models combine these results alongside data taken from observational studies to predict the molecular abundances in interstellar environments. This task is challenging due to missing measurements of many reaction rates and a lack of clarity on crucial parameters in processes such as diffusion, desorption, and reaction barriers (e.g. Cuppen et al. 2017; Penteado, Walsh \& Cuppen 2017; Wakelam et al. 2017). Moreover, physicochemical models are constrained by computational limits; not all details can be considered. Hence, different models are tailored to focus on selected physical or chemical processes, while making simplified assumptions on other parts.

In this work, three physicochemical models are analysed. These models have already been published in Wakelam et al. (2014) for model W14-M20 [based on Aikawa et al. 2008, last published in Manigand et al. (2020)], Drozdovskaya et al. (2016) for model D16 and Stéphan et al. (in preparation) for model S20, and will be referred to as such in the text. Two out of the three models, W14-M20 and S20, are 1D and constructed specifically for IRAS 16293-2422 (hereafter, IRAS 16293), while D16 represents a more generic 2D approach to the formation of a low-mass protostar. Located in $\rho$ Ophiuchus, the system is composed of two sources, A and B. Recently, Maureira et al. (2020) have shown that source A is itself a close binary. The B component that is targeted by the $1 \mathrm{D}$ models has an estimated mass of $0.1 \mathrm{M}_{\odot}$ (Jacobsen et al. 2018). Sources A and B were targeted by the Protostellar Interferometric Line Survey (PILS; Jørgensen et al. 2016) executed with ALMA (Atacama Large Millimeter Array) and have shown to exhibit a variety of molecules, including COMs (e.g. Ligterink et al. 2017; Drozdovskaya et al. 2018; Jørgensen et al. 2018; Calcutt et al. 2019; Manigand et al. 2019).

This work assesses whether source-tailored modelling is needed to explain the observed molecular abundances around young stellar objects or if, and to what extent, generic models can improve our understanding of the chemistry in the earliest stages of star formation. The physical conditions and the abundances of simple, most abundant molecules based on these three physicochemical models are compared. These models consider and target different components of the evolving protostellar system and use different methods to calculate the physical parameters. After establishing the discrepancies between the calculated chemical outputs, the calculations are redone with the same chemical model for all three sets of physical input parameters. This eliminates the differences arising from the chemical networks and gives direct access to the differences stemming solely from the physical models.

Section 2 gives a summary of the physical and the chemical models. Section 3 presents the comparison of the physical models and describes the obtained molecular abundances with the respective chemical codes and then with the same model, followed by the discussion in Section 4. The conclusions are raised in Section 5 .
Table 1. Physical precollapse parameters for the individual models.

\begin{tabular}{lccc}
\hline Parameter & $\mathrm{W} 14-\mathrm{M} 20^{\mathrm{a}}$ & $\mathrm{S} 20^{\mathrm{b}}$ & $\mathrm{D} 16^{\mathrm{c}}$ \\
\hline$T_{\text {dust, gas }}(\mathrm{K})$ & 10 & 8 & 10 \\
$\mathrm{n}_{\mathrm{H}}\left(\mathrm{cm}^{-3}\right)$ & $2.0 \times 10^{4}$ & $2.3 \times 10^{4}$ & $4.0 \times 10^{4}$ \\
$\mathrm{~A}_{\mathrm{V}}(\mathrm{mag})$ & 4.5 & $5.0-7.5$ & 10 \\
$\zeta_{\mathrm{CR}}\left(\mathrm{s}^{-1}\right)$ & $1.3 \times 10^{-17}$ & $1.3 \times 10^{-17}$ & $5.0 \times 10^{-17}$ \\
$t_{\text {precollapse }}(\mathrm{yr})$ & $1.0 \times 10^{6}$ & $1.0 \times 10^{6}$ & $3.0 \times 10^{5}$ \\
$t_{\text {collapse }}(\mathrm{yr})$ & $3.4 \times 10^{5}$ & $3.2 \times 10^{5}$ & $2.5 \times 10^{5}$ \\
$t_{\text {*birth }}(\mathrm{yr})$ & $2.5 \times 10^{5}$ & $3.1 \times 10^{5}$ & $2.0 \times 10^{4}$ \\
$t_{\text {*birth }}\left(\mathrm{t}_{\text {collapse }}\right)$ & 0.73 & 0.97 & 0.08 \\
\hline
\end{tabular}

${ }^{a}$ Physical model from Wakelam et al. (2014), based on Aikawa et al. (2008), last published in Manigand et al. (2020); ${ }^{b}$ Stéphan et al. (in preparation); ${ }^{c}$ last published in Drozdovskaya et al. (2016).

\section{MODELS}

Here, a short summary of the physical and chemical models used to obtain the results is given. All models assume $T_{\text {dust }}=T_{\text {gas }}$.

\subsection{Physical models}

\subsubsection{W14-M20}

This 1D model follows the evolution of a hydrostatic, prestellar core (Masunaga \& Inutsuka 2000) to a protostellar object under the assumption of free-fall collapse and has been used to model the envelope of IRAS 16293 (Aikawa et al. 2008, most recently updated in Wakelam et al. 2014). The prestellar core is characterized by an atomic $\mathrm{H}$ density $\left(\mathrm{n}_{\mathrm{H}}\right)$ of $2.0 \times 10^{4} \mathrm{~cm}^{-3}$, a visual extinction $\left(\mathrm{A}_{\mathrm{V}}\right)$ of $4.5 \mathrm{mag}$, a cosmic ray ionization rate $\left(\zeta_{\mathrm{CR}}\right)$ of $1.3 \times 10^{-17} \mathrm{~s}^{-1}$, and gas and dust temperatures $\left(T_{\text {dust }}\right)$ of $10 \mathrm{~K}$ (see Table 1$)$. These parameters are kept constant for $t_{\text {precollapse }}^{\mathrm{W} 14-\mathrm{M} 20}=1.0 \times 10^{6} \mathrm{yr}$ and then are evolved for $t_{\text {collapse }}^{\mathrm{W} 14-\mathrm{M} 20}$. Compressional heating leads to an accumulation of a central density, and $560 \mathrm{yr}$ before the protostellar birth, the first hydrostatic core (FHC) is formed. Only recently a candidate FHC has been observed Karnath et al. (2020). Theory suggests FHC formation occurs as soon as the central density is high enough $\left(\sim 10^{13} \mathrm{~g} \mathrm{~cm}^{-3}\right)$ for the inner region to become opaque to radiation (Dunham et al. 2014). The FHC is characterized by a radius of 1 au in this model. When its density increases to $10^{7} \mathrm{~g} \mathrm{~cm}^{-3}$ and the temperature to $2000 \mathrm{~K}$, a protostar is born at $2.5 \times 10^{5} \mathrm{yr}$ into the collapse $\left(=t_{* \mathrm{birth}}^{\mathrm{W} 14-\mathrm{M} 20}\right.$ in Table 1$)$. Thereafter, the model follows the evolution for an additional $9.3 \times 10^{4} \mathrm{yr}$. As the observed densities obtained from single-dish multiwavelength dust and molecular observations of the envelope of the IRAS 162932422 system by Crimier et al. (2010) are about 10 times larger than the ones given by the model, the calculated densities are multiplied by this factor at all times and radii (Wakelam et al. 2014; Andron et al. 2018; Coutens et al. 2019; Manigand et al. 2020). Model W14-M20 assumes that the core is embedded in an ambient cloud. As a consequence, the visual extinction of the prestellar phase is increased by three magnitudes to the given value of 4.5 mag (Aikawa et al. 2008). During collapse, the model by Aikawa et al. (2008) calculates the visual extinction via the column density of hydrogen nuclei $\left(\mathrm{N}_{\mathrm{H}}\right)$ from the outer core edge to the position of each parcel via $\mathrm{A}_{\mathrm{V}}=\mathrm{N}_{\mathrm{H}} /\left(1.59 \times 10^{21} \mathrm{~cm}^{-2}\right)$ mag. Thus, the attenuating column of material is that from the outer envelope shell to the position of a parcel at time $t$. No additional radiative transfer solver is used for the calculation of the radiation field nor the temperature distribution. The temperature is computed parametrically according to the model of Masunaga \& Inutsuka (2000). This model considers an external 
UV field, which includes surrounding stars and CRs that produce FUV photons through excitation of $\mathrm{H}_{2}$. The model only considers sufficiently dense parts of the envelope, where the influence of the internal UV flux from the emerging protostar is assumed to be negligible. Three representative trajectories that end up at distances of $15,62.4$, and 125 au from the protostar are considered in this work to trace the physical evolution of collapsing material.

\subsection{2 $S 20$}

The S20 model is presented in Stéphan et al. (in preparation) and is tailored towards IRAS 16293B. The initial physical conditions of the precollapse phase are $n_{\mathrm{H}}=2.3 \times 10^{4} \mathrm{~cm}^{-3}, T_{\text {dust }}=8 \mathrm{~K}, A_{\mathrm{V}}=5.0$ $7.5 \mathrm{mag}$ (depending on the radial distance from the protostar), and $\zeta_{\mathrm{CR}}=1.3 \times 10^{-17} \mathrm{~s}^{-1}$ and are evolved for $t_{\text {precollapse }}^{\mathrm{S} 20}=1.0 \times 10^{6} \mathrm{yr}$ (Table 1). Assuming free-fall collapse, the collapsing envelope is traced by tracking the position of gas parcels for $3.2 \times 10^{5} \mathrm{yr}$, the considered duration of the collapse. The density profile is derived from observations by Schöier et al. (2002) and is assumed to equal the density profile at the end of the collapse. This is used to calculate the power-law density profiles of the individual trajectories. The derived parameters of the best-fitting model in Schöier et al. (2002) set the radius of the inner envelope to $32 \mathrm{au}$, which equals the final position of the innermost trajectory. The accretion model for the protostar is taken from Hosokawa \& Omukai (2009), where the accretion rate $\left(\dot{M}_{*}\right)$ is kept at $10^{-5} \mathrm{M}_{\odot} \mathrm{yr}^{-1}$ during the collapse. The luminosity of the central protostar $\left(L_{*}\right)$ is assumed to be $10.5 \mathrm{~L}_{\odot}$ based on the protostellar accretion model of Hosokawa \& Omukai (2009), which turns on at $t_{* \mathrm{birth}}^{\mathrm{S} 20}=3.1 \times 10^{5} \mathrm{yr}$. Under the assumption that $T_{\text {gas }}=T_{\text {dust }}$ at all times, the dust temperature profiles of the trajectories are calculated with the radiative transfer code RADMC3D (Dullemond et al. 2012). The $F_{\mathrm{UV}}$ field is set to the arbitrary value of $10^{-8} \mathrm{G}_{0}$, where $\mathrm{G}_{0}$ is $1.6 \times 10^{-3} \mathrm{erg} \mathrm{cm}^{-2} \mathrm{~s}^{-1}$, at all times throughout the collapse. This work considers trajectories that end at distances of 32, 49.7, 61.9, 101, 125, 163, 203, and $232 \mathrm{au}$.

\subsubsection{D16}

The D16 model assumes an axisymmetric, semi-analytic collapse in $2 \mathrm{D}$ and tracks the physical evolution of the system including disc formation. This dynamic collapse model is adapted from Shu (1977) and further developed by Visser et al. (2009), Visser \& Dullemond (2010), Visser, Doty \& van Dishoeck (2011), and Harsono et al. (2013). Starting with the precollapse parameters $T_{\text {dust }}=10 \mathrm{~K}$, $n_{\mathrm{H}}=4.0 \times 10^{4} \mathrm{~cm}^{-3}, \mathrm{~A}_{\mathrm{V}}=10 \mathrm{mag}$, and $\zeta_{\mathrm{CR}}=5.0 \times 10^{-17} \mathrm{~s}^{-1}$ that are evolved for $t_{\text {precollapse }}^{\mathrm{D} 16}=3.0 \times 10^{5} \mathrm{yr}$, the collapse proceeds for $2.5 \times 10^{5} \mathrm{yr}$ (Table 1). Adapted from Young \& Evans (2005), the model forms its FHC during the first $2.0 \times 10^{4} \mathrm{yr}$ of the collapse. The radius of the FHC is estimated to be 5 au (Masunaga \& Inutsuka 2000) in this model. As the collapse continues, it transitions down to its initial protostellar radius of $\sim 2.5 \mathrm{R}_{\odot}$, following the calculations of Palla \& Stahler (1991) within $<100 \mathrm{yr}$ independent of other parameters (Visser et al. 2009). Hence, at $2.0 \times 10^{4} \mathrm{yr}, R_{*}$ equals the radius calculated by Palla \& Stahler (1991). As soon as the decrease of the radius stops, the protostar is born. At early times, the protostellar luminosity is driven by shock accretion. At later times, in the pre-main sequence phase of stellar evolution, the luminosity stems from gravitational contraction and deuterium burning (based on D’Antona \& Mazzitelli 1994; Visser et al. 2009). The now premain sequence star continues to grow by accreting mass from the forming disc and the infalling envelope. The model is evolved until it reaches its so-called accretion time ( $t_{\text {acc }}$, for this work: $\left.t_{\text {collapse }}\right)$, which is defined as the end of the primary accretion phase on to the star At this point, the outer shell of the envelope has travelled inwards to reach the protoplanetary disc. This depends on the initial parameters that are chosen to solve the hydrodynamic equations of a collapsing isothermal sphere (Shu 1977). The parameters include the initial core mass, which is set to $1 \mathrm{M}_{\odot}$, the gravitational constant $(\mathrm{G})$, the effective sound speed $\left(\mathrm{c}_{\mathrm{s}}\right)$ and a constant $m_{0}=0.975$ that stems from the analytical solution of the collapse model (Shu 1977). Thus, this model traces the evolution after the stellar birth for $2.3 \times 10^{5} \mathrm{yr}$. At all times during this evolution, 2D density and velocity distributions are obtained. To compute the temperatures $\left(T_{\text {dust }}=T_{\text {gas }}\right)$ and the stellar radiation field, the results of the collapse are fed into the radiative transfer code RADMC-3D (Dullemond et al. 2012). This model does not account for sources of external UV, because it assumes that the star-forming system is deeply embedded in a core. However, it includes the protostar after $t_{* \text { birth }}$ as a source of internal UV and takes CRs, which produce FUV photons through excitation of $\mathrm{H}_{2}$ into account. The resulting $F_{\mathrm{UV}}$ flux as calculated by RADMC-3D can be converted to the visual extinction $\left(A_{\mathrm{V}}\right)$ via the scaling relation:

$A_{\mathrm{V}}=\tau_{\mathrm{UV}, \mathrm{eff}} / 3.02$,

where $\tau_{U V \text {, eff }}$ is the effective UV extinction (Bohlin, Savage \& Drake 1978) calculated with

$\tau_{\mathrm{UV}, \text { eff }}=-\ln \left(\frac{F_{\mathrm{UV}}}{\pi \times \int_{\mathrm{F}_{\mathrm{UV}}} B_{\lambda}\left(T_{*}\right) \mathrm{d} \lambda \times \mathrm{R}_{*}^{2} /\left(R^{2}+z^{2}\right)}\right)$.

The denominator equals the blackbody radiation over the $F_{\mathrm{UV}}$ wavelength range from 912 to $2066 \AA$ (corresponding to 6.0$13.6 \mathrm{eV}$ ) with geometrical dilution, $\pi$ accounts for the radiation stemming from one hemisphere towards a point in the envelope (Drozdovskaya et al. 2015). The stellar radius is denoted as $R_{*}, R$ is the radial distance from the protostar, and $z$ describes the scale height. Here, the column of attenuating material is that between a position $(R, z)$ at time $t$ and the centre of the system, which is where the radiating emerging protostar is located.

Two disc cases are discussed in detail in Drozdovskaya et al. (2014, 2016). For this work, the case denoted as 'infall-dominated disc', or also 'case 7', is used. As can be seen in fig. 5 of Drozdovskaya et al. (2014), this model results in an extended, massive disc $\left(R_{\text {disc }}\right.$ $\sim 300$ au and $M_{\text {disc }} \sim 0.44 \mathrm{M}_{\odot}$ at $t_{\text {collapse }}^{\text {D16 }}$ ) with densities up to $\sim 10^{12} \mathrm{~cm}^{-3}$ in the mid-plane in the proximity of the protostar. The resulting disc is cold, with dust temperatures ranging between $\sim 20$ and $100 \mathrm{~K}$. Dust temperatures $>150 \mathrm{~K}$ are obtained in the outflow cavities and the most inner regions. The protostellar mass at $t_{\text {collapse }}^{\mathrm{D} 16}$ equals $\sim 0.56 \mathrm{M}_{\odot}$. For this work, the trajectories at 10.8, 20.6, 30.2, 40.2, and 46.7 au from Drozdovskaya et al. (2016) are investigated.

\subsection{Chemical models}

In this section, a brief description of the chemical models that are used to calculate the molecular abundances is given. For detailed discussions of the included mechanisms the reader is referred to the respective publications: Ruaud, Wakelam \& Hersant (2016) for W14-M20, Garrod (2013) for S20, and Walsh et al. (2014) for D16. Table 2 lists the considered reaction mechanisms of these chemical codes; the individual subsections describe their specific details.

In the three chemical models studied in this work, gaseous and solid phases are considered. The solid phase corresponds to the icy mantles that cover the dust grains. In the case of three-phase models (S20 and W14-M20), the icy mantle is further partitioned into a bulk and a surface layer. In the subsequent Sections 3.23.5 , the three physicochemical models will be compared in terms 
Table 2. Considered reaction mechanisms in the three chemical codes.

\begin{tabular}{|c|c|}
\hline Chemical phase & Reaction mechanism \\
\hline Gas phase & $\begin{array}{l}\text { Two-body associations (typical: neutral- } \\
\text { neutral reactions, ion-molecule reactions) } \\
\text { Photodissociation }^{a} \\
\text { Direct cosmic ray (CR) ionization }\end{array}$ \\
\hline Gas-grain interactions & $\begin{array}{l}\text { Thermal desorption } \\
\text { Non-thermal desorption: } \\
\text { Photodesorption }{ }^{\mathrm{a}} \\
\text { Reactive desorption } \\
\text { Spot-heating by CRs } \\
\text { Adsorption }\end{array}$ \\
\hline Solid phase & $\begin{array}{l}\text { Two-body associations (typical: radical- } \\
\text { Radical associations via thermal } \\
\text { Hopping and/or quantum tunneling) } \\
\text { Photodissociation }^{\text {a }}\end{array}$ \\
\hline
\end{tabular}

$\overline{{ }^{a} \text { Includes all sources of UV photons included in the specific model: stellar }}$ and interstellar UV photons, internally generated UV photons produced by the de-excitation via fluorescence cascades of $\mathrm{H}_{2}$ molecules excited by $\mathrm{CR}$ impacts.

of simple, most abundant molecules. The chosen species represent molecules common to the majority of existing chemical codes of pre- and protostellar cores that are frequently observed in different environments of the ISM. The nine molecules considered are carbon monoxide $(\mathrm{CO})$, carbon dioxide $\left(\mathrm{CO}_{2}\right)$, water $\left(\mathrm{H}_{2} \mathrm{O}\right)$, formaldehyde $\left(\mathrm{H}_{2} \mathrm{CO}\right)$, methanol $\left(\mathrm{CH}_{3} \mathrm{OH}\right)$, methane $\left(\mathrm{CH}_{4}\right)$, ammonia $\left(\mathrm{NH}_{3}\right)$, molecular nitrogen $\left(\mathrm{N}_{2}\right)$, and hydrogen sulphide $\left(\mathrm{H}_{2} \mathrm{~S}\right)$. In this work, atomic abundances are given relative to $\mathrm{n}_{\mathrm{H}}$ and molecular abundances relative to $\mathrm{n}_{\mathrm{H}_{2}}$.

In all physicochemical models, the chemistry is calculated in two steps. First, under constant precollapse physical parameters (Table 1) the initial atomic abundances (Table A1) are evolved for $t_{\text {precollapse }}$. The obtained molecular abundances (Table A2) are then used as initial input for the second step: the modelling of the collapse phase under its changing physical conditions.t

\subsubsection{NAUTILUS}

NAUTILUS is a three-phase chemical code used with W14-M20. The bulk and the surface vary in chemical reactivity. The two outermost ice monolayers correspond to the surface. Exchange between the bulk and the surface layers occurs via swapping, which describes the transport of each individual species from the bulk to the surface and vice versa; the net transfer rate equals 0 . The swapping rate from the bulk to the surface depends on the amount of layers in the bulk. Thermal and non-thermal desorption, and accretion are considered only for the surface. Photodissociation and diffusion are taken into account for the surface and the bulk, but the diffusion rates for the bulk species are kept smaller: the diffusion barrier for the surface is set to $E_{\mathrm{diff}}^{\mathrm{s}}=0.4 \times E_{\mathrm{des}}$ (desorption energy), while for the bulk $E_{\mathrm{diff}}^{\mathrm{b}}=0.8 \times E_{\mathrm{des}}$ is used. Recent dedicated theoretical efforts of Shingledecker et al. (2019) corroborate the lack of bulk diffusion at low temperatures. Moreover, solid-phase reactions are allowed in both ice components. No direct interactions between the gas phase and the bulk are considered. The Langmuir-Hinshelwood mechanism is the sole grain-surface reaction mechanism that is used (Ruaud et al. 2016). The rate equation approach for gas interactions and grain-surface chemistry is adapted from Hasegawa, Herbst \& Leung (1992) and Hasegawa \& Herbst (1993). The chemical network of Ruaud et al. (2016) has recently been extended by Manigand et al. (2020) to include larger COMs.

\subsubsection{MAGICKAL}

To calculate the molecular abundances with the S20 model, the three-phase model MAGICKAL (Model for Astrophysical Gas and Ice Chemical Kinetics and Layering; Garrod 2013) was used. MAGICKAL uses also the rate equation approach (Hasegawa et al. 1992; Hasegawa \& Herbst 1993) for gas and grain-surface reactions; and considers active chemistry in the gas phase, the icy surface of the dust particles, and the bulk ice. COM formation up to glycine is considered. In total, the chemical network includes 1369 species and over 21000 reactions. As for NAUTILUS, the swapping mechanism between the surface and the bulk produces no net transfer between the two phases. The swapping barrier is set to $E_{\text {swap }}=0.7 \times E_{\text {des }}$. For the most important species, individual $x$ in $E_{\mathrm{diff}}=x \times E_{\mathrm{des}}$ are tabulated (table 4 in Garrod 2013). As for NAUTILUS, accretion from the gas on to the surface, and thermal and non-thermal desorption from the surface are allowed. No direct reactions between the bulk and the gas take place; the bulk material must first be transferred to the surface. Solid-phase reactions, diffusion, and photodissociation are allowed for the surface and the bulk. Reaction-diffusion competition is accounted for via activation-energy barriers. Quantum tunnelling is allowed and the Languir-Hinshelwood is the only grainsurface reaction mechanism (Garrod 2013). The tunnelling process is adapted from Hasegawa et al. (1992), where it is parametrized by an expression for tunnelling through a rectangular potential. The barrier width is estimated from calculations (Garrod \& Pauly 2011).

\subsubsection{D16}

Details about the chemical code in the D16 model can be found in Drozdovskaya et al. (2014), Walsh et al. (2014), Drozdovskaya et al. (2016), and the references therein. Contrary to the other two models, this model treats the bulk and the surface as one equally chemically active phase (i.e. a two-phase model). The included gasphase network is the RATE12 release of the UMIST Database for Astrochemistry (UDfA; McElroy et al. 2013). The rate equation approach by Hasegawa et al. (1992) and Hasegawa \& Herbst (1993) is adapted for gas and grain-surface reactions, as well as their quantum tunneling parametrization. Again, quantum tunneling is allowed, the Langmuir-Hinshelwood mechanism is the sole mechanism for grain-surface chemistry (Walsh et al. 2014). Photodissociation and photoionization induced by the UV field are included in the network. Multiple families of COMs are included in the network. For all reactions $E_{\text {diff }}$ is set to $0.3 \times E_{\text {des }}$. A coverage factor included in the calculation of the photodesorption rate assures that photodesorption occurs only in the top two monolayers of the surface, as suggested by experiments (Bertin et al. 2012).

\section{RESULTS}

\subsection{Physical parameters}

Physical parameters as calculated by the three physical models are depicted in Fig. 1. While discussing the different physical parameters the inequality of the time-scales should be kept in mind. In the case of D16, no time-steps after $2.5 \times 10^{5} \mathrm{yr}$ are considered, as the accretion time has elapsed by this point. In the other two models, the protostars have not been born yet by this time. This occurs only after $2.5 \times 10^{5} \mathrm{yr}$ and $3.1 \times 10^{5} \mathrm{yr}$ for W14-M20 and S20, respectively, with the evolution being traced for $3.4 \times 10^{5} \mathrm{yr}$ and $3.2 \times 10^{5} \mathrm{yr}$ in total, respectively. 

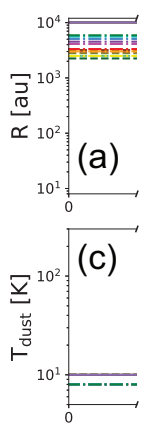
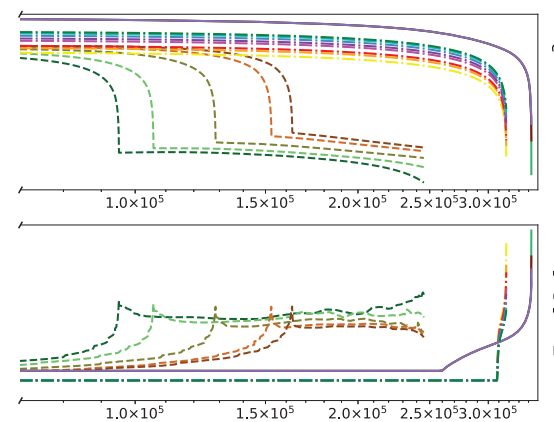

$\mathrm{t}_{\text {collapse }}[\mathrm{yr}$
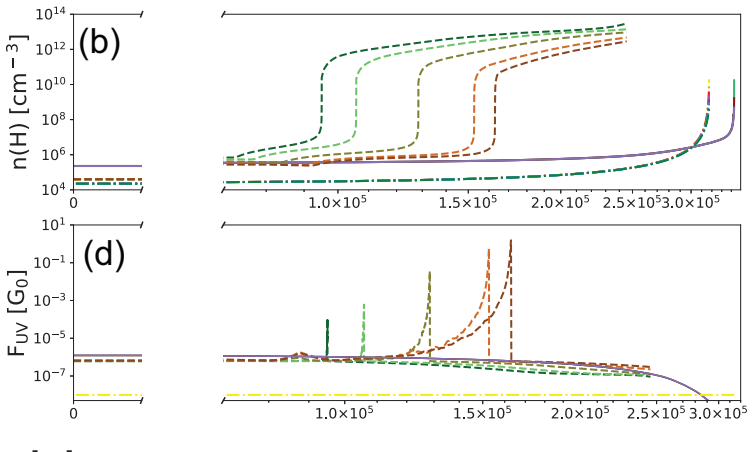

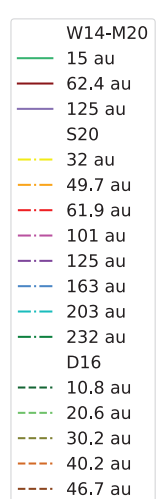

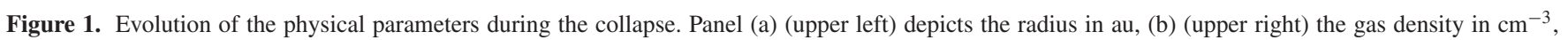

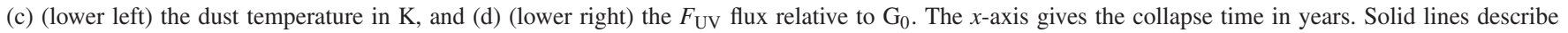
W14-M20, dotted-dashed S20, and dashed D16 with the colours corresponding to different final positions of the trajectories.

\subsubsection{Radial position}

Panel (a) traces the distance to the central source during the collapse. All trajectories emerge from far out in the envelope; their infall paths start between $\sim 2000-6000$ au for S20, 2000-3000 au for D16, and $\sim 10000$ au for W14-M20. The infall pathways show that the material remains in the outer envelope at distances beyond 1000 au for $\sim 2.8 \times 10^{5} \mathrm{yr}\left(\sim 0.88 t_{\text {collapse }}^{\text {S20 }}\right)$ for S20 and $3.4 \times 10^{5} \mathrm{yr}$ $\left(\sim 0.99 t_{\text {collapse }}^{\mathrm{W} 14-\mathrm{M} 20}\right)$ for $\mathrm{W} 14-\mathrm{M} 20$. For S20, the innermost trajectory ends at the assumed inner envelope boundary at 32 au (Schöier et al. 2002). Even at such proximity to the protostar this model never probes the material of the protoplanetary disc, because a disc is not considered. For W14-M20, the innermost trajectory at 15 au should also be in the protoplanetary disc regime, but the disc is not modelled in this case either. In the case of D16, the innermost trajectory breaks into the inner $100 \mathrm{au}$, which corresponds to the disc in this model, after $\sim 8.0 \times 10^{4} \mathrm{yr}\left(0.33 t_{\text {collapse }}^{\text {D16 }}\right)$. All considered trajectories follow this behaviour, the outermost trajectory reaches the innermost $100 \mathrm{au}$ after $\sim 1.6 \times 10^{5} \mathrm{yr}\left(0.66 t_{\text {collapse }}^{\mathrm{D} 16}\right)$. The $2 \mathrm{D}$ approach of this model also gives information about the scale height (z). At the end of the collapse, the considered trajectories reside in the mid-plane at $z \sim 0.01$ au (Drozdovskaya et al. 2016).

\subsubsection{Density}

Fig. 1(b) shows the evolution of the gas density for all three models. D16 results in the highest densities in the range of $10^{11}-10^{12} \mathrm{~cm}^{-3}$. These high values stem from a final location in the protoplanetary disc close to the mid-plane ( $z \sim 0.01 \mathrm{au}$; Drozdovskaya et al. 2016). As indicated by the evolution of the distance to the protostar, D16 enters a higher density range soon after the onset of the collapse $\left(\sim 8.0 \times 10^{4} \mathrm{yr} ; 0.33 t_{\text {collapse }}^{\mathrm{D} 16}\right)$. The sooner the trajectories travel inwards, the earlier an increase in density occurs. After the collapse, the innermost trajectory at 10.8 au shows the highest density of $\sim 3.4 \times 10^{12} \mathrm{~cm}^{-3}$, the outermost trajectory at 46.7 au the lowest of $\sim 4.2 \times 10^{11} \mathrm{~cm}^{-3}$. This behaviour is not reproduced in the W14-M20 and S20 models, as no protoplanetary disc is considered in these models. The gas density of W14-M20 stays constant during the majority of the collapse, no variation depending on the final distance to the protostellar source is seen. After $3.0 \times 10^{5} \mathrm{yr}\left(\sim 0.87 \mathrm{t}_{\text {collapse }}^{\mathrm{W} 14-\mathrm{M} 20}\right)$, the final values of around $\sim 10^{9} \mathrm{~cm}^{-3}$ are approached. This indicates that materials spend most of the collapse phase in the envelope and would only breach into the protoplanetary disc, if it were to be included in the model, at the very end. Similar behaviour is also seen for S20: it takes almost $3.0 \times 10^{5} \mathrm{yr}\left(\sim 0.95 t_{\text {collapse }}^{\mathrm{S} 20}\right)$ for the density to increase to $10^{6} \mathrm{~cm}^{-3}$. The final values of the considered trajectories range between $10^{8}$ and $10^{9} \mathrm{~cm}^{-3}$ depending on their distance to the protostar.

\subsubsection{Dust temperature}

The contrasting infall paths of the three physical models are also reflected in the evolution of the dust temperature as depicted in Fig. 1(c). The dust temperatures in D16, starting from $10 \mathrm{~K}$, reach their individual maxima around the time when the trajectories breach the innermost $100 \mathrm{au}$. The innermost trajectory arrives in proximity of the protostar earlier than others, and hence its $T_{\text {dust }}$ peaks first The temperatures of the considered trajectories at the end of the collapse range between $\sim 20$ and $60 \mathrm{~K}$, which is consistent with their positions in the cold, shielded mid-plane at $z \sim 0.01$ au (Drozdovskaya et al. 2016). At the end of the collapse, all have $T_{\text {dust }}$ lower than the maxima encountered at disc entry, except for the innermost parcel of material. The initial dust temperature in W14M20 is $10 \mathrm{~K}$ for all trajectories at the beginning of the collapse. Given the low-density regimes and the large radii, the temperatures remain low for the majority of the collapse. Warmer surroundings $(\sim 20-50 \mathrm{~K})$ are encountered as the emerging protostar heats up the encompassing material after $2.5 \times 10^{5} \mathrm{yr}\left(=t_{* \mathrm{birth}}^{\mathrm{W} 14-\mathrm{M} 20}\right)$. Close to the end of the collapse the innermost trajectory reaches values of $\sim 270 \mathrm{~K}$, resulting in the highest temperatures of all considered trajectories in this work. The dust temperatures calculated with S20 are the lowest of the considered models and share an initial value of $8 \mathrm{~K}$, which remains constant for the majority of the collapse. After the protostar has turned on after $\sim 3.1 \times 10^{5} \mathrm{yr}\left(=\mathrm{t}_{* \mathrm{birth}}^{\mathrm{S} 20}\right)$, an increase in temperature is seen. Even though this model remains in the low-temperature $(8 \mathrm{~K})$ regime for the longest time, the final temperatures are higher than those in D16 (ranging from 30 to $210 \mathrm{~K}$ depending on the proximity to the protostar). Thus, contrasting to D16, $T_{\text {dust }}$ at the end of the collapse equals the temperature maxima. Fig. 1 shows that the distance to the protostar does not correlate with the dust temperature, but rather with the density regime for all models. Although D16's trajectories end up closest to the protostar, the calculated $T_{\text {dust }}$ values from S20 and W14-M20 are higher by an order of magnitude (S20 yields a dust temperature of $\sim 210 \mathrm{~K}$ for the trajectory at $32 \mathrm{au}$, while D16 has $\sim 25 \mathrm{~K}$ at 30.2 au due to the proximity to the mid-plane). The dust temperature correlates with 
distance to the protostar only as long as the material remains in the envelope with a smooth, radial density profile.

\subsection{4 $F_{U V}$ flux}

The $F_{\mathrm{UV}}$ flux is shown in Fig. $1(\mathrm{~d})$ relative to $\mathrm{G}_{0}$. For $\mathrm{S} 20$, the $\mathrm{F}_{\mathrm{UV}}$ field is assumed to be $10^{-8} \mathrm{G}_{0}$ at all points in time. The $F_{\mathrm{UV}}$ flux in D16 is obtained from calculations with RADMC-3D, as detailed in Section 2.1.3. In this model, the individual $F_{\mathrm{UV}}$ maxima of the trajectories correlate with the infall of the material closer to the protostellar source, where the gas density and the dust temperature show a significant increase. All trajectories remain at a constant value of $\sim 10^{-6} \mathrm{G}_{0}$ for the first $\sim 8.0 \times 10^{4} \mathrm{yr}\left(0.33 t_{\text {collapse }}^{\text {D16 }}\right)$. Afterwards, $F_{\text {UV }}$ peaks at individual points in time with the innermost trajectory peaking first at the lowest maximum $F_{\mathrm{UV}}$. The two outermost trajectories reach $\sim 1 \mathrm{G}_{0}$, due to their entry into the disc being later in time, in comparison to the innermost trajectories, and the protostellar luminosity increasing with time (fig. 2 of Drozdovskaya et al. 2014). Afterwards, a decline occurs for all trajectories towards the end of the collapse upon their entry into the shielded protoplanetary disc. Calculations of the $F_{\mathrm{UV}}$ flux are not included in model W14M20, but $A_{\mathrm{V}}$ is calculated (Section 2.1.1). In order to compare the models, the $F_{\mathrm{UV}}$ flux shown in Fig. 1 is obtained via the expression $F_{\mathrm{UV}}=F_{0} \exp \left(-\tau_{\mathrm{UV}, \text { eff }}\right)$ (Visser et al. 2011), where $\tau_{\mathrm{UV}, \text { eff }}$ is calculated with equation (1). $F_{0}$ is set to $1 \mathrm{G}_{0}$, which assumes an impinging unattenuated UV field of $1 \mathrm{G}_{0}$ at every position in the envelope. W14-M20 shows the highest $F_{\mathrm{UV}}$ flux at the beginning of the collapse with a value of $\sim 10^{-6} \mathrm{G}_{0}$ for all considered trajectories. A decrease in the flux is seen after $\sim 2.0 \times 10^{5} \mathrm{yr}\left(\sim 0.58 t_{\text {collapse }}^{\mathrm{W} 14-\mathrm{M} 20}\right)$. At the end of the collapse, the $F_{\mathrm{UV}}$ flux becomes negligible as the parcels are far from the external UV field. In comparison to the initial values, the fluxes at the end of the collapse decrease by about an order of magnitude for D16. Due to the disc entry a spike in the $F_{\mathrm{UV}}$ flux occurs for D16, but all trajectories end in the shielded environment of the disc. This decrease from initial to final also occurs for W14M20; however, for a different reason that is the distancing from the externally impinging UV radiation.

\subsection{Tracing the physical components in the models}

As discussed in Section 3.1, trajectories pass through various physical components of a low-mass star-forming region during their infall towards the central source. Depending on the model, this can include components such as the inner and outer envelopes, protoplanetary disc, and outflow cavities. Here, all three models are described in the context of the generic 2D D16 model.

\subsubsection{Tracing the physical components in the models}

Fig. 2 addresses the components of low-mass star formation (Section 3.2) encountered by three trajectories of the models studied in this work. The final positions of the trajectories considered are 46.7 au (D16), 49.7 au (S20), and 62.4 au (W14-M20) and are also later used to study the chemical evolution in detail. The radial position of the infalling trajectories is depicted as a function of gas density (left-hand panel) and dust temperature (right-hand panel). The depicted parameter ranges of the shaded regions are taken from the D16 model as detailed in fig. 5 and section 3.1.2 in Drozdovskaya et al. (2014) and resemble the description of the 2D model when the trajectory of 46.7 au enters the forming protoplanetary disc. The size of the protoplanetary disc is set to $130 \mathrm{au}$, but it extends further as the
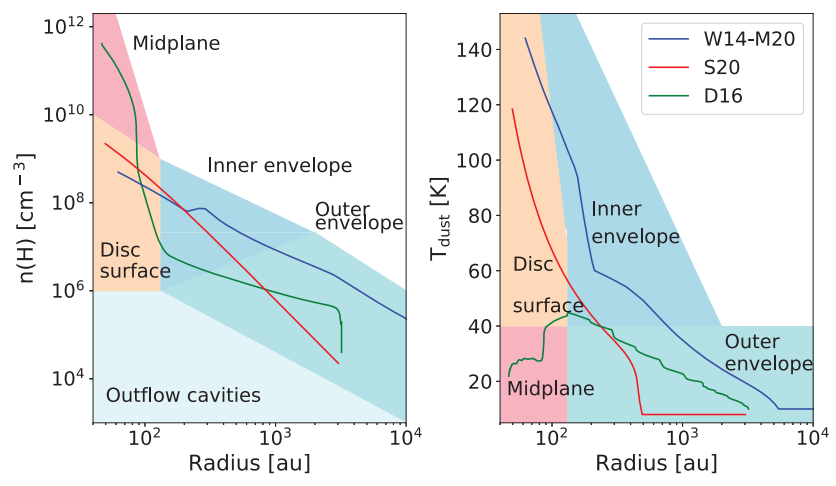

Figure 2. Gas density relative to $\mathrm{n}_{\mathrm{H}}$ in $\mathrm{cm}^{-3}$ (left-hand panel) and dust temperature regimes in Kelvin along the infall paths of the discussed trajectories as a function of distance to the protostar. The W14-M20 model is plotted in blue, S20 in red, and D16 in green. The shaded regions correspond to regions of a star-forming system.

system evolves. The outer envelope is characterized by $\mathrm{n}_{\mathrm{H}}=10^{6}-$ $10^{7} \mathrm{~cm}^{-3}$ and $T_{\text {dust }}<40 \mathrm{~K}$ with a radial distance beyond $130 \mathrm{au}$. The same radial distance, but densities between $10^{6}$ and $10^{9} \mathrm{~cm}^{-3}$ and $\mathrm{T}_{\text {dust }}>40 \mathrm{~K}$ are attributed to the inner envelope. The protoplanetary disc regime accounts for the disc surface and the mid-plane: the disc surface covers regions with $\mathrm{n}_{\mathrm{H}}$ from $10^{6}$ to $10^{10} \mathrm{~cm}^{-3}$ and $T_{\text {dust }}>40 \mathrm{~K}$, the mid-plane shows densities of at least $10^{9}-10^{10} \mathrm{~cm}^{-3}$ depending on the radial distance and $T_{\text {dust }}<40 \mathrm{~K}$. The outflow cavities can extend over the full considered radial distance of the model and show $n_{\mathrm{H}}$ between $10^{4}$ and $10^{6} \mathrm{~cm}^{-3}$ and $T_{\text {dust }} \sim 90-300 \mathrm{~K}$.

As shown by the different traced density and dust temperature regimes in Fig. 2, it becomes evident that the three models are constructed for different regions of the forming system. All models start their infall paths in the outer envelope, their infalls are accompanied by increasing $\mathrm{n}_{\mathrm{H}}$, as they pass the inner envelope and approach their final positions in the disc regime. The 1D nature of W14-M20 and S20 combined with being tailored towards IRAS 16293 results in very similar gas densities at their final positions $\left(\sim 10^{9} \mathrm{~cm}^{-3}\right)$. Accounting for the $2 \mathrm{D}$ structure of the system, D16 traces the trajectory as it approaches the mid-plane at $z \sim 0.01 \mathrm{au}$, which results in a gas density at the final position of the order of $10^{11} \mathrm{~cm}^{-3}$. Due to the deviating $t_{* \mathrm{birth}}$, the infalling material covers distinct distances unperturbed by the influence of the protostar. As soon as the models evolve past $t_{* \text { birth }}, T_{\text {dust }}$ starts to increase. D16 is already influenced at the beginning of its infall path. W14-M20 travels 5000 au before the protostar emerges. Afterwards, $T_{\text {dust }}$ increases up to $\sim 140 \mathrm{~K}$ at its final position. The steady increase is given due to the parametrization of $T_{\text {dust }}$ in terms of radius (Section 2.1.1). S20 lies only at a distance of $500 \mathrm{au}$ at $t_{* \text { birth }}$, then $T_{\text {dust }}$ increases to $\sim 120 \mathrm{~K}$. The final position in the shielded mid-plane for D16 is accountable for the $T_{\text {dust }}$ of $\sim 20 \mathrm{~K}$ at the end of the calculations, its temperature maximum of $\sim 45 \mathrm{~K}$ is obtained prior to the disc entry. $2 \mathrm{D}$ models allow a more comprehensive exploration of the different physical components of a star-forming system and cover a more complete range of encountered values of physical parameters.

\subsection{Initial atomic abundances}

The initial atomic abundances at the start of the prestellar core phase are plotted in Fig. 3 (values given in Table A1). In the case of W14-M20, C, S, Fe, and $\mathrm{Cl}$ are initially purely ionic. For this work, the most important elements are atomic $\mathrm{H}$ and molecular $\mathrm{H}_{2}$, and $\mathrm{C}, \mathrm{N}, \mathrm{O}$, and $\mathrm{S}$. The values for $\mathrm{C}, \mathrm{N}$, and $\mathrm{O}$ concur for 


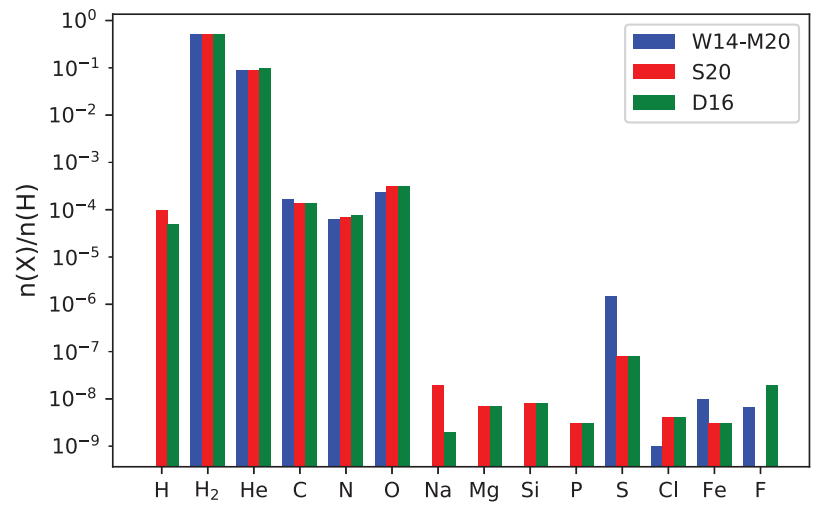

Figure 3. Initial atomic abundances (Table A1) at the start of the precollapse phase relative to $n_{H}$. Blue bars correspond to the values of the W14-M20 model, red bars depict the S20 model, and green bars refer to the D16 model. All species first start in the gas. In the case of W14-M20, C, S, Fe, and Cl are initially purely ionic.

all three models: their values differ by less than a factor of 10 . However, the initial abundance of sulphur differs by two orders of magnitude $\left(\mathrm{S}^{+} / \mathrm{n}_{\mathrm{H}} \sim 10^{-6}\right.$ for $\mathrm{W} 14-\mathrm{M} 20, \mathrm{~S} / \mathrm{n}_{\mathrm{H}} \sim 10^{-8}$ for D16 and $\mathrm{S} 20)$. Another difference between the models is the value of atomic hydrogen. While D16 contains $10^{-5}$, S20 begins with $10^{-4}$. In W14$\mathrm{M} 20$, all $\mathrm{H}$ is initially in its molecular form. The overall number of hydrogen nuclei $\left(n_{\mathrm{H} \text {,nuclei }}\right)$ is given by $n_{\mathrm{H} \text {,atomic }}+2 n_{\mathrm{H}_{2}}$. Availability of atomic hydrogen is important for hydrogenation reactions on the surfaces of grains. All models also include elements that are not used to produce the molecules central to this work. Fluorine is included only in W14-M20 and D16, Fe and Cl are present in all three models. Furthermore, $\mathrm{S} 20$ and D16 include $\mathrm{Na}, \mathrm{Mg}, \mathrm{Si}$, and $\mathrm{P}$, which all lie in the range of $10^{-8}-10^{-9}$ relative to $\mathrm{n}_{\mathrm{H}}$. In the case of $\mathrm{D} 16$, all values are taken from the UMIST data base, which account for typical abundances in dark cloud cores (see McElroy et al. 2013 for details). The values used by the $\mathrm{S} 20$ model with the exception of $\mathrm{H}$ and $\mathrm{H}_{2}$ have already been published in Garrod (2013).

\subsection{Precollapse molecular abundances}

The precollapse molecular abundances are plotted in Figs 4 and A1 (values listed in Table A2). To allow a proper comparison of the three-phase versus the two-phase chemical models, the abundances in the bulk and on the surface are summed to obtain the total abundances in the ice. Furthermore, to highlight chemical evolution rather than the partition between phases, the gas and ice abundances have been summed. Due to the cold dust temperatures $(\sim 10 \mathrm{~K})$ of the precollapse phase, the molecules in Fig. 4 are predominantly found as solids (Fig. A1). Good agreement between models is obtained for the hypervolatile (Bisschop et al. 2006) species $\mathrm{N}_{2}$ and $\mathrm{CO}$ (within a factor of $\sim 1.4-1.6$ ), and also for $\mathrm{H}_{2} \mathrm{O}$ and $\mathrm{CH}_{4}$ (factor of 1.12.6). In the case of $\mathrm{CO}_{2}$, two models (W14-M20 and D16) produce almost identical numbers (within a factor of 2), but S20 lies two orders of magnitude lower. This is a bit puzzling, as the species that play an important role in the formation of $\mathrm{CO}_{2}$ (specifically, $\mathrm{CO}$ and $\mathrm{H}_{2} \mathrm{O}$; Noble et al. 2011) show comparable numbers in all the models. $\mathrm{CO}_{2}$ forms on grain surfaces via the reaction of $\mathrm{CO}$ and $\mathrm{OH}$, with $\mathrm{OH}$ originating from the photodissociation of $\mathrm{H}_{2} \mathrm{O}$, when dust temperatures are sufficiently low to keep $\mathrm{CO}$ in the solid phase (Drozdovskaya et al. 2016). The underproduction of $\mathrm{CO}_{2}$ in S20 may be explained by the small overproduction of $\mathrm{H}_{2} \mathrm{O}$ and $\mathrm{CH}_{4}$ in that model, which decreases the availability of $\mathrm{C}$ and $\mathrm{O}$

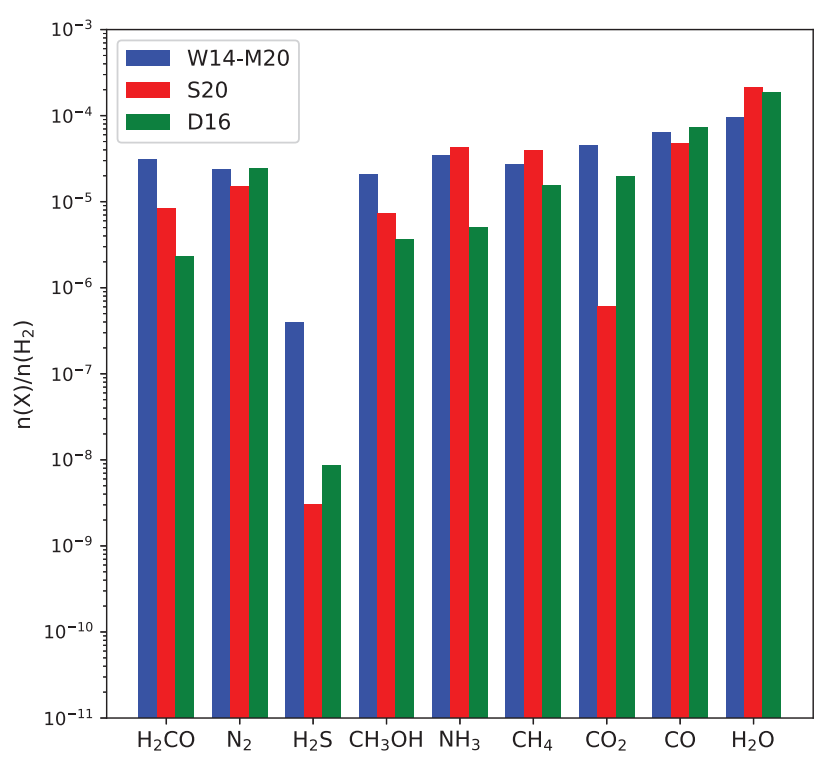

Figure 4. Precollapse molecular abundances (Table A2) at the start of the collapse phase relative to $\mathrm{n}_{\mathrm{H}_{2}}$. Blue bars describe the values of the W14-M20 model, red bars show the S20 model, and green bars correspond to the D16 model. This plot depicts the sum of gases and ices (individual phases are shown in Fig. A1).

to form $\mathrm{CO}_{2}$. Another possibility is the lack of UV in model S20, which limits the amount of $\mathrm{OH}$ stemming from the photodissociation of $\mathrm{H}_{2} \mathrm{O}$. Thus, less $\mathrm{OH}$ is available to form $\mathrm{CO}_{2}$ via the reaction of $\mathrm{CO}$ and $\mathrm{OH}$. For $\mathrm{NH}_{3}$, the abundance in D16 is an order of magnitude lower than in W14-M20 and S20. In the case of $\mathrm{H}_{2} \mathrm{CO}$, and $\mathrm{CH}_{3} \mathrm{OH}$, the values lie within the same order of magnitude, but with D16 having the lowest abundances. The D16 model has the shortest precollapse time-scale (Table 1), thereby reducing the amount of time for species such as $\mathrm{NH}_{3}, \mathrm{H}_{2} \mathrm{CO}$, and $\mathrm{CH}_{3} \mathrm{OH}$ to form via hydrogenation reactions (Ioppolo, Cuppen \& Linnartz 2011; Fedoseev et al. 2015). Consequently, $t_{\text {precollapse }}$ is a critical parameter for molecules formed in prestellar cores. However, if initial atomic abundances vary by more than an order of magnitude, then this will also affect the molecules formed. This is the reason for the big differences in the abundance of $\mathrm{H}_{2} \mathrm{~S}: \sim 10^{-7}$ (W14-M20) over $10^{-8}$ (D16) to $10^{-9}$ (S20) relative to $n_{H_{2}}$.

\subsection{Postcollapse molecular abundances}

In order to compare the chemical evolution during the collapse as computed by the three physicochemical models, one trajectory per model is selected. The final positions considered are 46.7 au (D16), 49.7 au (S20), and 62.4 au (W14-M20). Fig. 5 depicts the sum of the gas and ice abundances during the collapse along these three trajectories of the three physicochemical models analysed in this work. Figs B1-B9 contain the analogous figures for the two phases separately on a molecule by molecule basis.

\subsubsection{Similarities}

W14-M20 and S20 predominantly trace the chemistry in the envelope at large scales from the forming protostar. Fig. 5 shows that the prestellar abundances remain unaltered for the majority of the collapse (for $t<0.95 t_{\text {collapse }}^{\mathrm{S} 20}$ and $t<0.87 t_{\text {collapse }}^{\mathrm{W} 14-\mathrm{M} 20}$, respectively) with no chemical evolution of the material taking place. In comparison to 

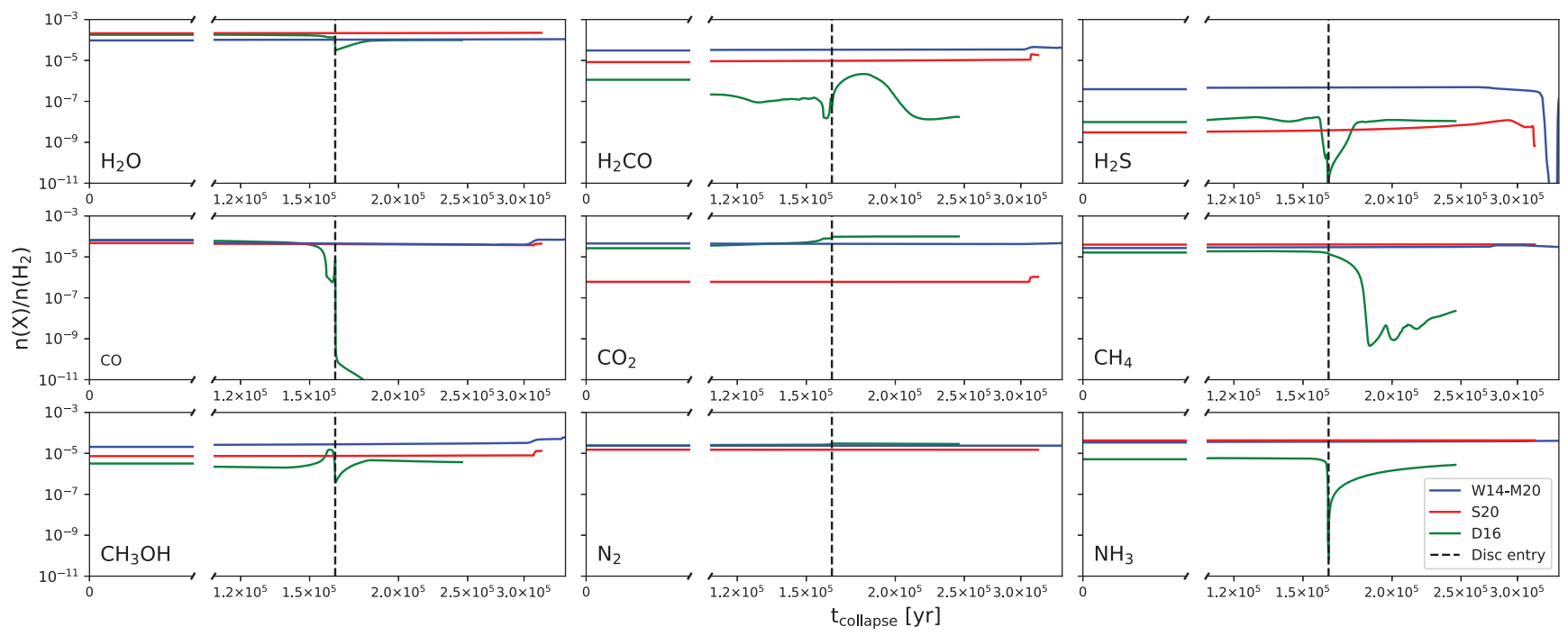

Figure 5. This figure depicts the sum of the abundances in the gas and ice relative to $\mathrm{n}_{\mathrm{H}_{2}}$ for all molecular species throughout the collapse. Blue represents W14-M20 (final position at $62.4 \mathrm{au}$ ), red S20 (final position at $49.7 \mathrm{au}$ ), and green D16 (final position at $46.7 \mathrm{au}$ ). The dashed black line marks the disc entry of the D16 model.

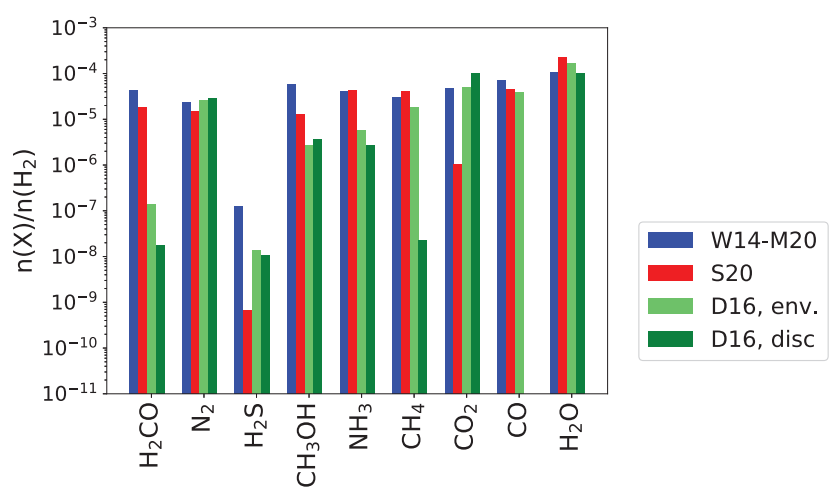

Figure 6. Postcollapse molecular abundances relative to $n_{H_{2}}$. Blue bars describe the values of the W14-M20 model (final position at $62.4 \mathrm{au}$ ), red bars show the S20 model (final position at $49.7 \mathrm{au}$ ), light green bars correspond to the abundances in the warm envelope upon disc entry in the D16 model (at $\left.t=1.5 \times 10^{5} \mathrm{yr}=0.61 t_{\text {collapse }}^{\mathrm{D} 16}\right)$, dark green bars illustrate the abundances in the disc at the end of the collapse in the D16 model (final position at $46.7 \mathrm{au}$ ).

D16, this is only reproduced for $\mathrm{N}_{2}$ and $\mathrm{H}_{2} \mathrm{O}$ (although, even $\mathrm{H}_{2} \mathrm{O}$ shows a relatively small response to the disc entry, Section 3.5.3).

\subsubsection{Differences arising from the precollapse molecular abundances}

At the onset of collapse the abundances of $\mathrm{H}_{2} \mathrm{~S}$ and $\mathrm{CO}_{2}$ differ by several orders of magnitude between the models (Section 3.4). These differences are preserved during the collapse and at the end (Figs 5 and 6). Both of these molecules do also undergo some additional chemical processing during the collapse, most noteworthy in D16. This is also seen for the case of $\mathrm{H}_{2} \mathrm{CO}$, but to a lesser extent. The abundance of $\mathrm{H}_{2} \mathrm{~S}$ is lowered at some point during the collapse in all models. In D16, it decreases by three orders of magnitude upon disc entry (Section 3.5.3) due to photodissociation, but it is reformed quickly once inside the disc up to its envelope abundance. In S20, $\mathrm{H}_{2} \mathrm{~S}$ begins to undergo destruction at times greater than $t_{* \text { birth }}$ due to photodissociation.
For all models, the evolution of the $\mathrm{CO}_{2}$ abundances display the same trend: it increases during the collapse (by a factor of $\sim 5$ for W14-M20, 75 for S20, and 400 for D16).

$\mathrm{H}_{2} \mathrm{CO}$ increases its abundance towards the end of the collapse for W14-M20 and S20, the difference between the models is an attribute of the abundance at the start of the collapse. While it undergoes additional processing in D16 (Section 3.5.3), its maximum throughout the collapse never exceeds its precollapse molecular abundance, which is about an order of magnitude lower than for the other two models as discussed in Section 3.4.

\subsubsection{Strong influence by the disc entry}

Given the 1D nature of W14-M20 and S20 representative of the envelope, the impact of the disc entry can only be assessed for D16. The trajectory enters the protoplanetary disc at $\sim 1.65 \times 10^{5} \mathrm{yr}$ $\left(\sim 0.67 t_{\text {collapse }}^{\text {D16 }}\right)$. The disc is characterized by cold $(<50 \mathrm{~K})$ dust temperatures, high densities $\left(>10^{9} \mathrm{~cm}^{-3}\right)$, and small radii $(<100 \mathrm{au})$. This results in a rapid change in physical conditions experienced by the infalling material from the envelope. Fig. 5 shows that the majority of the species are heavily impacted by the disc entry in D16: the changes seen in the abundances of $\mathrm{CO}, \mathrm{H}_{2} \mathrm{CO}$, and $\mathrm{CH}_{3} \mathrm{OH}$ are the result of hydrogenation reaction rates being strongly affected in the range of dust temperatures and densities experienced at the disc entry (Fuchs et al. 2009). Photodissociation due to the strong $F_{\mathrm{UV}}$ in the inner envelope causes a large decrease in the abundances of $\mathrm{NH}_{3}$ and $\mathrm{H}_{2} \mathrm{~S}$ prior to the disc entry. This is also the reason for the small change in the abundance of $\mathrm{H}_{2} \mathrm{O}$ (Section 3.5.1). This, in turn, leads to the production of $\mathrm{CO}_{2}$, making $\mathrm{CO}_{2}$ more abundant in D16 than in the other two physicochemical models (Section 3.5.2). The photostable $\mathrm{N}_{2}$ ( $\mathrm{Li}$ et al. 2013) is the only molecule that is not affected by the disc entry (Section 3.5.1).

\subsubsection{Young versus mature envelope}

Fig. 6 shows the abundances of nine selected molecules at the respective $t_{\text {collapse }}$ of the three physicochemical models, W14-M20, 
S20, and D16. As discussed in Section 3.5.3, the disc entry significantly alters the abundances of almost all molecules. Consequently, in Fig. 6, a comparison is also made to the envelope abundances of D16 that are obtained before the trajectory enters the disc. The envelope abundances of D16 are taken at an age of $1.5 \times 10^{5} \mathrm{yr}$ $\left(\sim 0.61 t_{\text {collapse }}^{\text {D16 }}\right)$, which implies that it is a younger envelope in comparison to the mature envelopes of W14-M20 and S20 at an

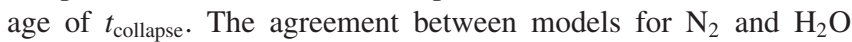
remains also for the envelope (Sections 3.5.1 and 3.5.3). $\mathrm{CO}_{2}$ and $\mathrm{H}_{2} \mathrm{~S}$ differences persist as a result of their initial precollapse abundances (Section 3.5.2). Some of the hydrogenation-dominated species (CO, $\mathrm{H}_{2} \mathrm{CO}, \mathrm{NH}_{3}$; Section 3.5.3) now show a closer agreement in the envelope within a factor of 2 for $\mathrm{CO}$ and $10^{2}$ for $\mathrm{H}_{2} \mathrm{CO}$ (compared to a factor of $10^{3}$ for the postcollapse abundance). $\mathrm{NH}_{3}$ now shows an agreement within a factor of 7 , as the envelope abundance is its abundance prior to photodissociation at disc entry (Section 3.5.3). The $\mathrm{CH}_{3} \mathrm{OH}$ abundance remains in close agreement between the models (with the envelope abundance being a mere factor of 1.4 lower than the abundance at $t_{\text {collapse }}$ ). Finally, $\mathrm{CH}_{4}$ also drastically improves in terms of agreement as the envelope abundance is almost three orders in magnitude higher than in the disc in D16. The younger age of the D16 envelope is the reason for the envelope abundances being systematically lower for hydrogenation-dominated species $\left(\mathrm{CO}, \mathrm{H}_{2} \mathrm{CO}, \mathrm{CH}_{3} \mathrm{OH}, \mathrm{NH}_{3}\right) . \mathrm{CO}_{2}$ is the only species more abundant in the envelope of D16 than in W14-M20 and most-notably in S20 due to significant $F_{\mathrm{UV}}$ flux in the inner envelope at lukewarm temperatures, which is not a regime covered in the other two models (Sections 3.5.1 and 3.5.2). Therefore, the envelope age is important for interpreting chemical outputs of physicochemical models, as well as the rate at which the physical parameters are evolved.

\subsection{Molecular abundances with the same chemical model}

Dismantling all three chemical networks that all include hundreds of species and thousands of reactions is not feasible. Instead, the molecular abundances are re-calculated with the two-phase chemical code of D16 for the precollapse physical parameters of W14-M20 and S20. Although the precollapse abundances are now calculated with the same chemical code, differences in the molecular budget at the onset of the collapse remain distinct due to the differences in the physical parameters. These are compared in Section 3.6.1. For the results presented in Section 3.6.2, the adopted prestellar physical parameters and abundances of the D16 model are taken as identical initial conditions for the W14-M20 and S20 models as well (Table A1). This approach allows tracking the difference in the chemical evolution solely based on the input provided by the physical model of the collapse phase.

\subsubsection{Precollapse molecular abundances}

The recalculations of the chemical evolution during the precollapse stage are depicted in Fig. 7. Calculations are performed for precollapse durations of $3.0 \times 10^{5} \mathrm{yr}$ and $1.0 \times 10^{6} \mathrm{yr}$ for all three models. While the individual physical precollapse parameters are implemented (Table 1), only the initial atomic abundances of the D16 model (Table A1) are considered.

Running the same chemical model for the same precollapse duration shows that the marginally different precollapse parameters still affect the outcome of the molecular abundances. Neither for a precollapse duration of $3.0 \times 10^{5} \mathrm{yr}$ (indicated by filled squares in Fig. 7) nor for a precollapse duration of $1.0 \times 10^{6} \mathrm{yr}$ (indicated

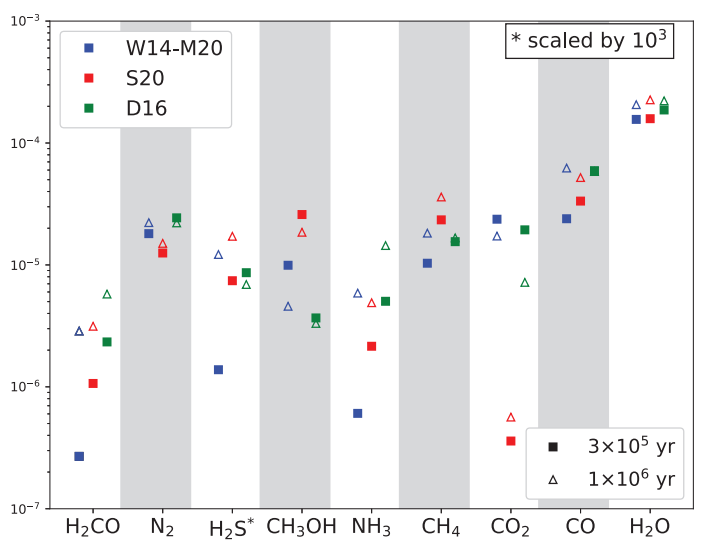

Figure 7. Precollapse molecular abundances at the start of the collapse phase relative to $\mathrm{n}_{\mathrm{H}_{2}}$ for the considered trajectories calculated with the chemical model of D16 (gases and ices have been summed). Blue markers describe model W14-M20, red markers present S20, and green markers depict D16. The fiducial D16 model for $t_{\text {precollapse }}^{\mathrm{D} 16}=3.0 \times 10^{5} \mathrm{yr}$ is indicated by the filled green squares. Filled blue and red squares are used to describe W14M20 and S20 for the same precollapse duration. Triangle markers describe a precollapse duration of $1.0 \times 10^{6} \mathrm{yr}$. Note that the abundances of $\mathrm{H}_{2} \mathrm{~S}$ are scaled by a factor of $10^{3}$ for all models.

by hollow triangles in Fig. 7), is a perfect match obtained for any single molecule. This shows that prestellar density, dust temperature, and extinction values are critical to the highest precision. In turn, the precollapse duration is the most critical physical parameter. While the spread between the models for the same duration is significant, the abundance differences for different durations can be orders of magnitude apart. With the exception of $\mathrm{CH}_{3} \mathrm{OH}$, the longer precollapse duration leads to a higher abundance of the species formed via hydrogenation reactions on the grain surfaces $\left(\mathrm{CH}_{4}, \mathrm{NH}_{3}, \mathrm{H}_{2} \mathrm{O}\right.$, and $\left.\mathrm{H}_{2} \mathrm{CO}\right)$. This hints that COM formation already occurs efficiently during the precollapse phase. Production of $\mathrm{H}_{2} \mathrm{CO}, \mathrm{CH}_{3} \mathrm{OH}$, and $\mathrm{COMs}$ via efficient hydrogenation explains the reduction of $\mathrm{CO}_{2}$ for $t_{\text {precollapse }}=10^{6} \mathrm{yr}$ for models D16 and W14-M20. The longer precollapse duration also increases the CO abundances for S20 and W14-M20, in the case of D16 the value does not change.

\subsubsection{Postcollapse molecular abundances}

For the calculation of the chemical evolution during the collapse, the precollapse molecular abundances of D16 (Table A2) are used as a starting point to remove the influence of the different abundances at $t_{\text {collapse }}=0$. The chemical evolution during the collapse as calculated with the chemical code of D16 for trajectories of all the physical models considered in this work is depicted in Fig. 8 with solid lines. As a reference for the reader, the dashed lines correspond to the original results obtained with NAUTILUS and MAGICKAL for the W14M20 and S20 trajectories, respectively. Recalculating the chemical evolution allows to split the molecular abundances into two distinct groups.

\subsubsection{Variations mainly due to the now-identical adopted precollapse abundances}

Fig. 8 shows that the evolution of $\mathrm{N}_{2}, \mathrm{H}_{2} \mathrm{O}, \mathrm{CH}_{4}, \mathrm{NH}_{3}, \mathrm{H}_{2} \mathrm{~S}$, and $\mathrm{CO}_{2}$ are hardly impacted by the switch of the chemical network (less than a factor of 10). This is also true for CO in S20. The 

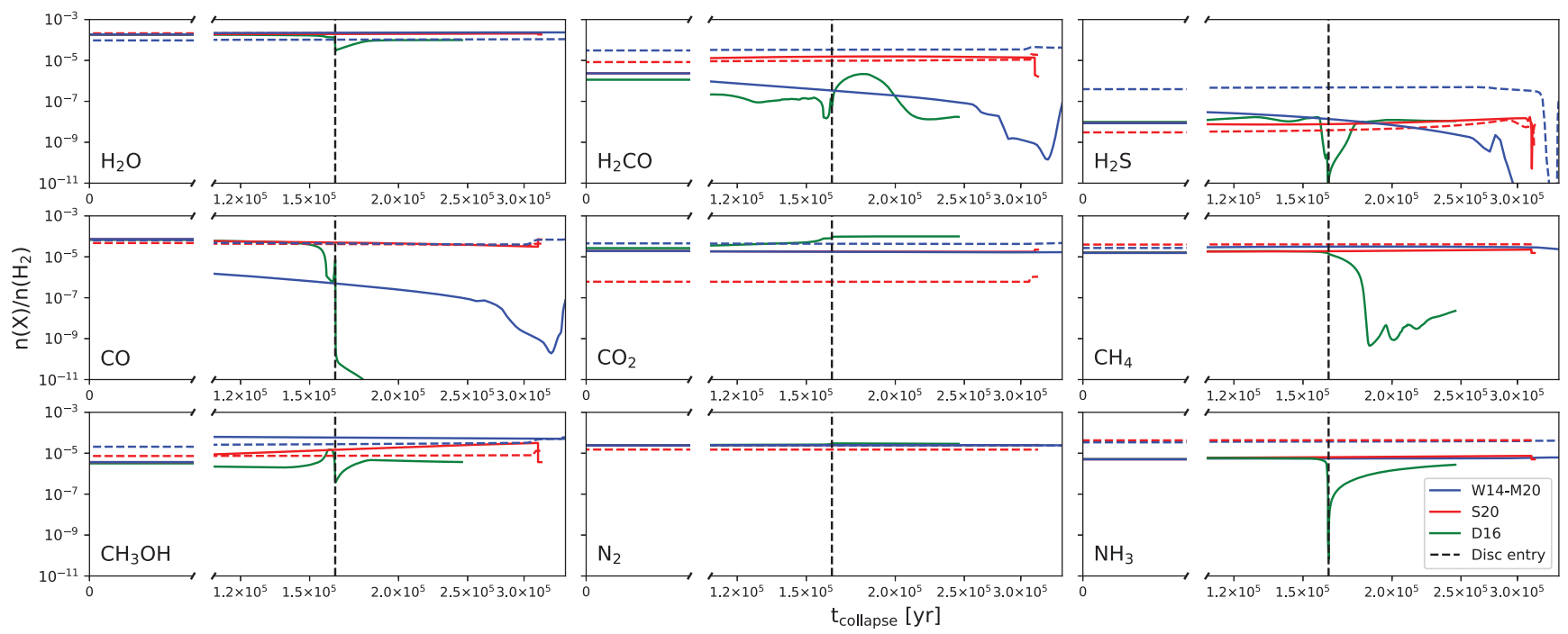

Figure 8. This figure depicts the sum of the abundances in the gas and ice relative to $\mathrm{n}_{\mathrm{H}_{2}}$ for all molecular species throughout the collapse. Blue represents W14-M20 (final position at $62.4 \mathrm{au}$ ), red S20 (final position at $49.7 \mathrm{au}$ ), and green D16 (final position at $46.7 \mathrm{au}$ ). The dashed black line marks the disc entry of the D16 model. The solid lines represent results obtained with the two-phase chemical network. The dashed lines correspond to results from NAUTILUS (blue) and MAGICKAL (red) and are depicted as a reference.

observed shift in abundances between the old and new calculations stems only from the now different molecular abundances at the beginning of the collapse. This shows that neither the newly adopted binding energy of $0.3 \times E_{\mathrm{des}}$ (in contrast to $0.35 \times E_{\mathrm{des}}$ for S20 and $0.4 \times E_{\mathrm{des}}$ for W14-M20) nor the different number of reactants and reactions influence these molecules at cold conditions before $t_{* \text { birth }}$ and after, as they enter the hot corino and encounter warmer regions with higher gas densities. For these species, the abundance during the collapse can be computed accurately with two- and three-phase chemical models once the initial precollapse abundance is set.

\subsubsection{Variations due to the choice of the chemical network}

Major differences between the results obtained with the two-phase and three-phase chemical models arise in species that are critical in the $\mathrm{CO}$ hydrogenation sequence towards $\mathrm{H}_{2} \mathrm{CO}$ and $\mathrm{CH}_{3} \mathrm{OH}$. In comparison to the three-phase calculations, the following results are obtained with the two-phase computation: the $\mathrm{CO}$ abundance is lower for S20 and W14-M20; the $\mathrm{H}_{2} \mathrm{CO}$ abundance is higher for S20 and lower for W14-M20; the $\mathrm{CH}_{3} \mathrm{OH}$ abundance is higher for W14M20 and S20. For the case of S20 (MAGICKAL), the differences are typically less than one order of magnitude. However, for the case of W14-M20 (NAUTILUS), the differences for $\mathrm{CO}$ and $\mathrm{H}_{2} \mathrm{CO}$ can be as large as three orders of magnitude. The relatively small differences between the two-phase model and S20 (MAGICKAL) suggest that two-phase models do not necessarily overproduce reactivity in the solid phase. This is supported by recent findings of Sipilä, Caselli \& Taquet (2016), which show that bulk ice models are better suited to describe the chemistry in starless cores. The source of the large discrepancies between models may stem from adopted activation barriers in individual reactions. These are then carried forwards throughout the entire duration of the collapse. Differences seen in Fig. 6 between the postcollapse abundances of the three models are reduced, as expected, upon computation with an identical chemical network (Fig. B10).

\section{DISCUSSION}

\subsection{Time-scales}

For the three studied models, the precollapse phase lasts either $3 \times 10^{5} \mathrm{yr}$ (D16) or $10^{6} \mathrm{yr}$ (W14-M20 and S20; Table 1). Dynamical evolution is proposed for cloud cores in the turbulent paradigm with a lifetime of $1-10$ free-fall times $\left(t_{\mathrm{ff}}\right)$ :

$t_{\mathrm{ff}}=\sqrt{\frac{3 \pi}{32 G \rho}}=\sqrt{\frac{3 \pi}{32 G m_{\mathrm{H}} \mu_{\mathrm{p}}\langle n\rangle}}$,

where $G$ is the gravitational constant, $m_{\mathrm{H}}$ is the mass of the hydrogen atom, $\mu_{\mathrm{p}}$ is the mean molecular weight per particle, and $\langle n\rangle$ is the average gas density (André, Basu \& Inutsuka 2009). For the models considered in this work, $t_{\mathrm{ff}}$ spans between $\sim 1.7 \times 10^{5} \mathrm{yr}\left(t_{\mathrm{ff}}^{\mathrm{D} 16}\right)$ and $3.17 \times 10^{5} \mathrm{yr}\left(t_{\mathrm{ff}}^{\mathrm{S} 20}\right)$. Thus, the precollapse time-scales of $3.0 \times 10^{5}$ $\left(\sim 1.8 t_{\mathrm{ff}}^{\mathrm{D} 16}\right)$ and $1.0 \times 10^{6} \mathrm{yr}\left(\sim 3.2 t_{\mathrm{ff}}^{\mathrm{S} 20}\right)$ lie well within the theoretical $1-10 t_{\mathrm{ff}}$ range.

Observations of dense cores indicate that their lifetimes depend on mass. Studies of isolated cores have shown that their lifetimes as starless cores decrease as the density increases (Jessop \& WardThompson 2000). Observations of Lee, Myers \& Tafalla (1999) suggest that the typical lifetime of a starless core with an average density of $\sim 10^{4} \mathrm{~cm}^{-3}$ should be of the order of $1.0-1.5 \times 10^{6} \mathrm{yr}$. This is longer than the time-scale used in D16, but the initial density in this model is also higher than $10^{4} \mathrm{~cm}^{-3}$. Observations of prestellar cores in Perseus, Serpens, and Ophiucus show that the ratio of starless to protostellar cores in each cloud is close to unity, which suggests that the core lifetime before the onset of collapse should be similar to the lifetime of the embedded protostar. This leads to an average prestellar lifetime of $\sim 4.5 \times 10^{5} \mathrm{yr}$ with an uncertainty of a factor of 2 , which is also in good agreement with the models presented here if one assumes that all starless cores eventually will turn prestellar (Enoch et al. 2008).

Even though the different $t_{\text {precollapse }}$ of the models all lie within an acceptable range of the observations and theory, the difference of $7.0 \times 10^{5} \mathrm{yr}$ still impacts the precollapse molecular budget 
(Figs 4 and A1; Section 3.4). Another relevant time-scale is $t_{\text {collapse, }}$, which is set to $2.5 \times 10^{5} \mathrm{yr}(\mathrm{D} 16), 3.4 \times 10^{5} \mathrm{yr}(\mathrm{W} 14-\mathrm{M} 20)$, and $3.2 \times 10^{5} \mathrm{yr}(\mathrm{S} 20)$. Observations find that the collapse, if turbulence is the primary driver, should last 1-2 $t_{\mathrm{ff}}$ (Enoch et al. 2008). This is in agreement with $\mathrm{W} 14-\mathrm{M} 20$ and $\mathrm{S} 20$, as their respective $t_{\text {collapse }}$ equal $1 t_{\text {eff }}$ (S20) or fall in the theoretically determined range of $1-2 t_{\mathrm{ff}}\left(\sim 1.44 t_{\mathrm{ff}}^{\mathrm{W} 14-\mathrm{M} 20}\right)$. The D16 model $\left(t_{\text {collapse }}^{\mathrm{D} 16} \sim 1.46 t_{\mathrm{ff}}^{\mathrm{D} 16}\right)$ also fits the observational evidence, even though this model does not assume free-fall collapse (Section 2.1.3). However, quantifying the contraction motions and thus the dynamical evolution can only be achieved by carrying out a detailed radiative transfer study. This allows to compare computed line profiles with observational ones (e.g. Keto \& Caselli 2008 for prestellar cores). Furthermore, it is to note that $t_{* \text { birth }}$ is close to $t_{\mathrm{ff}}$ for W14-M20 $\left(\sim 0.95 t_{\mathrm{ff}}^{\mathrm{W} 14-\mathrm{M} 20}\right)$ and $\mathrm{S} 20\left(\sim 0.97 t_{\mathrm{ff}}^{\mathrm{S} 20}\right)$, but this is not the case for D16 $\left(\sim 0.12 t_{\mathrm{ff}}^{\mathrm{D} 16}\right)$. The moment of stellar birth determines the onset of active chemistry during the process of star formation (Section 3.5.4) stimulated by higher dust temperatures and enhanced $F_{\mathrm{UV}}$ fluxes.

\subsection{Physical components of low-mass star-forming regions}

It is not easy to disentangle the envelope and the disc of young embedded protostars in observations. Traditionally, protostars are classified by their spectral energy distribution (SED; Lada 1987). The earliest Class 0 stage is deeply embedded, which makes observations at near-infrared (NIR) wavelengths difficult. Class I objects are still embedded in an envelope that feeds the protoplanetary disc and accretes on to the protostar, but these objects are more easily accessible in the NIR. In Class II objects, the envelope has dissipated, but the protoplanetary disc still accretes on to the protostar. Observations suggest that this phase sets in $\sim 5 \times 10^{5} \mathrm{yr}$ after the collapse (Dunham et al. 2014). The presence of the protostellar envelope at earlier times does not yet allow a direct comparison of discs associated with Class I to Class II objects for a big sample. Estimates from observations attribute radii as large as $>250$ au to them (Dunham et al. 2014; Li et al. 2014). This is consistent with D16 $\left(R_{\text {disc }} \sim 300\right.$ au at $\left.\mathrm{t}_{\text {collapse }}\right)$. In Class 0 objects, 90 percent of the NIR emission stems from the envelope. Consequently, if a disc-like structure exists at this stage, its characterization is very complicated. In recent years, high spatial resolution observations performed with ALMA have shown that COMs do already exist in very young discs (e.g. Codella et al. 2019; Lee et al. 2019). The increasing availability of data for the inner parts of protostellar systems will allow us to understand the detailed structures of these sources at different scales. Lifetime estimates of the Class 0 objects from observations suggest that this phase lasts between 1.5 and $1.6 \times 10^{5} \mathrm{yr}$ (Dunham et al. 2014). As the envelope is present until the end of the calculations in all models, while the age is greater than $1.6 \times 10^{5} \mathrm{yr}$, the modelled systems correspond to Class I objects at $t_{\text {collapse }}$ following the observational classification. Theoretical work of Robitaille et al. (2006) classified evolutionary stages according to the accretion rates from the envelope and the disc on to the protostar. This formalism is adapted for D16 by Harsono et al. (2013): Stage 0 is characterized by $M_{\text {env }}>>M_{*}\left(t / t_{\text {collapse }} \leq 0.5\right)$, Stage I by $M_{\text {env }}<M_{*}$, but $M_{\text {env }}>M_{\text {disc }}\left(t / t_{\text {collapse }}>0.5\right)$, and Stage II by $M_{\text {env }}<M_{\text {disc }}$, which puts all models into the Stage I category at the end of the calculations.

\subsection{Influence of the physical conditions on the chemical model}

Given the similarities of models S20 and W14-M20 (Fig. 1), the differences when using the same chemical model should not be as prominent as in Fig. 8, if the role of the physical conditions would not be critical. While the difference in values for, e.g. gas densities or dust temperatures might seem small and be well within the error limits, this will impact the results obtained with chemical networks on timescales applicable to star-forming systems. Cold temperatures at early stages allow gas-phase species to freeze out on to the dust grains, which is further enhanced by a high gas density. The decreasing abundances of gaseous species (e.g. CO) change the composition of the gas and thus the chemistry, as they become available for chemical processes on the grain surfaces instead. Theoretical and experimental work by Cuppen et al. (2009) and Fuchs et al. (2009) on the hydrogenation process from $\mathrm{CO}$ to $\mathrm{CH}_{3} \mathrm{OH}$ show that the obtained abundances and formation efficiencies of methanol and its intermediate products vary significantly within the considered narrow temperature range of $12-20 \mathrm{~K}$ and long interstellar timescales. As these variations are already significant in a system with a small number of species and possible reactions, their significance should not be underestimated in a large chemical network.

Another parameter that can influence the chemical evolution and thus should not be neglected is the CR ionization rate. Pioneering modelling work has been performed by Padovani et al. (2016), where the first comprehensive theoretical model of CR production and acceleration along protostellar jets and outflows is presented. Most molecular clouds are thought to have an ionization rate that varies spatially and can locally be enhanced by a few orders of magnitude as suggested by observations of, e.g. Ceccarelli et al. (2014), Podio et al. (2014), Fontani et al. (2017), and Favre et al. (2018). This has been reproduced by semi-empirical modelling work (Gaches, Offner \& Bisbas 2019). However, most models assume a standard CR ionization rate, which is the case for S20 and W14-M20. Moreover, CRs can penetrate the inner, dense parts, where species freeze out on to the dust grains that are shielded from interstellar $F_{\mathrm{UV}}$ and protostellar FUV and X-ray photons. While these species cannot thermally desorb due to the cold temperatures, CRs can hit dust grains and return these species into the gas phase via non-thermal desorption processes and localized thermal events (spot heating; Leger, Jura \& Omont 1985). CRs can also interact with $\mathrm{H}_{2}$ and produce FUV photons, which can subsequently lead to photodesorption from the grain surfaces (Öberg, van Dishoeck \& Linnartz 2009a; Öberg et al. 2009c) and photodissociation in the gas and directly in the ice. Furthermore, it has been shown that $\mathrm{CR}$ interactions can increase the temperature inside the cloud and consequently influence the gas-phase chemistry (Bisbas et al. 2017). Non-thermal chemistry induced by CRs has also been shown to produce COMs under cold core conditions (Shingledecker et al. 2018). In addition, CRs are believed to control the fractional abundance of hydrogen atoms in molecular clouds, which are then again important for, e.g., hydrogenation reactions on grain surfaces. The atomic $\mathrm{H}$ abundance can be explained if $\mathrm{H}_{2}$ is dissociated by CRs. As shown by Padovani et al. (2018), secondary electrons stemming from the primary ionization via CRs are the only source of atomic hydrogen at column densities representative of these environments. Either these secondary processes, a variable $\mathrm{CR}$ ionization rate as a result of $\mathrm{CR}$ attenuation, or spatially differing $\mathrm{CR}$ ionization rates will therefore impact the results of a chemical model. This is especially true if one considers that some models, as it is in the case of W14-M20, only account for molecular hydrogen, $\mathrm{H}_{2}$, at the beginning of the calculations.

Lastly, the considered time-scales have shown to play the dominant role in the outcome of chemical models. While the duration of the precollapse phase and duration prior to the birth of the protostar have already been discussed in Section 4.1, one further caveat is present in all three discussed models and common to astrochemical models in general: the chemical evolution is traced throughout the 
collapse phase and then a few $\times 10^{5} \mathrm{yr}$ at most after the birth of the protostar. These results are then used to infer the history of observed systems. However, it is unclear what the appropriate collapse time for each system is, it may be shorter or longer than the model time. Furthermore, it is hard to quantify the age of these systems accurately, and consequently, it is difficult to constrain how long the abundances after $t_{* \text { birth }}$ should be traced with the models.

\subsection{On the need for generic models}

Physicochemical models can be a powerful predictive tool. In preparation for observations, they can deliver valid estimates of the expected chemistry if the physical conditions of the region of interest are well constrained. On the other hand, they make use of molecular inventories obtained from observations to predict the evolutionary history of the system. The results presented in the previous sections show that this can be achieved with different modelling approaches. Both 1D and 2D approaches to the physical modelling part, and twophase and three-phase approaches to the chemical part derive similar results if they probe comparable physical conditions for the majority of the species considered in this work. However, 1D models do oversimplify the physical environment, even if some of the physical input, for example, the gas density in the case of W14-M20, or the envelope model for S20, are inferred from observations of a specific source. For observations with a low spatial resolution, where the majority of the probed material corresponds to the envelope; and the hot corino region is not resolved sufficiently; an adequate fit can still be obtained. However, in recent years facilities such as ALMA offer the possibility to constrain the structure of star-forming systems on smaller scales. It is questionable, if a simplified assumption about the structure of the system will lead to a reasonable constraint on the chemical evolution and the physical history of the system on all scales. Even the earliest phases of protostellar systems, Class 0 protostars, prior to the emergence of vivid protoplanetary discs, show evidence for multiple components. The CALYPSO survey targeted 16 Class 0 protostars, of which only 2 displayed Keplerian rotation, but for 11 the dust continuum was better reproduced by a model with a disc-like component (Maret et al. 2014, 2020). These results are further supported by investigation of the specific angular momentum profiles in the inner envelope of the targets (Gaudel et al. 2020). ALMA data from recent years complicates this picture further, as it suggests that the structures of protostellar sources are significantly more complicated than assumed by 1D and 2D models (e.g. Maureira et al. 2020). Efforts in modelling the chemistry with 3D magnetohydrodynamic (MHD) simulations (e.g. Hincelin et al. 2016; Zhao, Caselli \& Li 2018) also need to be considered. Thus, 1D models considering infall pathways purely within a cold, dense envelope are unlikely to produce representative chemical abundances in hot corinos surrounding protostars, even if some observational constraints are applied.

This should not lessen the importance of improving and testing the chemical networks. Included reactions rates need to be carefully evaluated and in many cases, data or its accuracy are lacking (for example, even for the well-studied hydrogenation of $\mathrm{CO}$ differences exist in the investigated models, Section 3.6.2). Furthermore, chemical networks are limited by computational capacities, which induces potential bottlenecks. If this is then coupled to a simplified assumption in the physical model, it is impossible to allocate from where differences between theoretical predictions and observational data arise. However, if no data of a source of interest is available to place constraints on the physical parameters, a simplified assumption is a valid approach to better constrain the physical parameters that can play a role in the formation or destruction of some species. None the less, including multiple components in the physical model, whether derived from observations for specific sources or in a generic approach to predict the chemical evolution over a physical parameter range should improve our understanding of crucial chemical processes during the various stages of star formation.

\section{CONCLUSIONS}

This work investigates different physicochemical modelling approaches to low-mass star formation. Benefits and caveats of using a simplified $1 \mathrm{D}$ or a more generic $2 \mathrm{D}$ physical model alongside two-phase or three-phase chemical networks have been discussed based on abundant, simple molecular species. While these models are powerful tools to derive the chemical evolution of these systems and investigate its initial formation conditions, seemingly small deviations of the input parameters can lead to significant differences in the output over astrophysical time-scales. The major findings are raised as follows:

(i) Assumptions about the precollapse phase need to be drawn carefully. Small differences in the physical parameters have shown to lead to significant deviations if evolved over the lifetime of a core. Instead of adopting standard literature values, a wider range of physical parameters and the consequent range of abundances should be taken into account.

(ii) The precollapse duration impacts not only the initial molecular budget at the beginning of the collapse (i.e. that of a prestellar core), but also the subsequent chemical evolution of the star-forming system. Even within applied constraints from observations of preand protostellar systems, the deduced results vary significantly. Disagreements are exacerbated if the initial elemental abundances vary by more than an order of magnitude.

(iii) The more advanced two-phase approach to modelling the surface chemistry does not impact the presented results much. For simple molecular species, no strong differences in abundances arise from the implementation of a two- or three-phase model for similar physical environments. Observations cannot differentiate between surface and bulk ice, which makes the accurate representation of the solid phase under the diverse interstellar conditions challenging in models.

(iv) A simplified physical model can be suitable for a first estimate to draw conclusions based on observations of low spatial resolution, when the underlying structures are missed. However, deviations between physicochemical models and observations should not always be attributed to caveats in the chemical network by default. Whether source-tailored or not, mismatch may stem from the assumption of a more simplified physical structure.

(v) High spatial resolution observations of inner regions of lowmass star-forming systems that probe the disc regime must be coupled with more sophisticated models that represent the relevant structures. The chemical composition of such systems can be computed accurately only if the duration of the collapse and the timing of protostellar birth can be constrained. Furthermore, radiative transfer molecular line simulations of contracting dense cores need to be carried out to place constraints on the dynamical evolution and time-scales relevant for chemistry.

Generic models with more comprehensive physics may not provide the optimal match to observations, but allow a source to be studied in perspective of other star-forming regions. Future observations on even small scales will require more detailed physicochemical models. 


\section{ACKNOWLEDGEMENTS}

The authors thank the referee P. Caselli for her helpful comments and suggestions that improved the paper. BMK and MND acknowledge the Swiss National Science Foundation (SNSF) Ambizione grant no. 180079. MND also acknowledges the Center for Space and Habitability (CSH) Fellowship, and the IAU Gruber Foundation Fellowship. AC acknowledges financial support from the Agence Nationale de la Recherche (grant no. ANR-19-ERC7-0001-01). SM acknowledges support from the H2020 European Research Council (ERC) (grant agreement no. 646908) through ERC Consolidator Grant 'S4F'. Research at Centre for Star and Planet Formation is funded by the Danish National Research Foundation. GS thanks T. Hosokawa for providing his stellar model results in digital form. GS also thanks R. Garrod for support, and the department of chemistry at UVA for funding the project.

\section{DATA AVAILABILITY}

The data underlying this article are available in the article.

\section{REFERENCES}

Agúndez M., Marcelino N., Cernicharo J., Roueff E., Tafalla M., 2019, A\&A, 625, A147

Aikawa Y., Wakelam V., Garrod R. T., Herbst E., 2008, ApJ, 674, 984

André P., Basu S., Inutsuka S., 2009, The Formation and Evolution of Prestellar Cores, Cambridge University Press, Cambridge, UK, p. 254

Andron I., Gratier P., Majumdar L., Vidal T. H. G., Coutens A., Loison J.-C., Wakelam V., 2018, MNRAS, 481, 5651

Benson P. J., Myers P. C., 1989, ApJS, 71, 89

Bergin E. A., Tafalla M., 2007, ARA\&A, 45, 339

Bertin M. et al., 2012, Phys. Chem. Chem. Phys. (Incorporating Faraday Transactions), 14, 9929

Bisbas T. G., van Dishoeck E. F., Papadopoulos P. P., Szűcs L., Bialy S., Zhang Z.-Y., 2017, ApJ, 839, 90

Bisschop S. E., Fraser H. J., Öberg K. I., van Dishoeck E. F., Schlemmer S., 2006, A\&A, 449, 1297

Bohlin R. C., Savage B. D., Drake J. F., 1978, ApJ, 224, 132

Calcutt H. et al., 2019, A\&A, 631, A137

Ceccarelli C., Dominik C., López-Sepulcre A., Kama M., Padovani M., Caux E., Caselli P., 2014, ApJ, 790, L1

Chacón-Tanarro A. et al., 2019, A\&A, 622, A141

Codella C. et al., 2019, ACS Earth Space Chem., 3, 2110

Coutens A. et al., 2019, A\&A, 623, L13

Crapsi A., Caselli P., Walmsley C. M., Myers P. C., Tafalla M., Lee C. W., Bourke T. L., 2005, ApJ, 619, 379

Cridland A. J., Eistrup C., van Dishoeck E. F., 2019, A\&A, 627, A127

Crimier N., Ceccarelli C., Maret S., Bottinelli S., Caux E., Kahane C., Lis D. C., Olofsson J., 2010, A\&A, 519, A65

Cuppen H. M., van Dishoeck E. F., Herbst E., Tielens A. G. G. M., 2009, A\&A, 508, 275

Cuppen H. M., Walsh C., Lamberts T., Semenov D., Garrod R. T., Penteado E. M., Ioppolo S., 2017, Space Sci. Rev., 212, 1

D’Antona F., Mazzitelli I., 1994, ApJS, 90, 467

Drozdovskaya M. N. et al., 2018, MNRAS, 476, 4949

Drozdovskaya M. N., Walsh C., Visser R., Harsono D., van Dishoeck E. F., 2014, MNRAS, 445, 913

Drozdovskaya M. N., Walsh C., Visser R., Harsono D., van Dishoeck E. F., 2015, MNRAS, 451, 3836

Drozdovskaya M. N., Walsh C., van Dishoeck E. F., Furuya K., Marboeuf U., Thiabaud A., Harsono D., Visser R., 2016, MNRAS, 462, 977

Dullemond C. P., Juhasz A., Pohl A., Sereshti F., Shetty R., Peters T., Commercon B., Flock M., 2012, Astrophysics Source Code Library, record ascl:1202.015
Dunham M. M. et al., 2014, in Beuther H., Klessen R. S., Dullemond C. P., Henning T., eds, Protostars and Planets VI. The University of Arizona Press, Tucson, AZ, USA, p. 195

Enoch M. L., Evans Neal J. I., Sargent A. I., Glenn J., Rosolowsky E., Myers P., 2008, ApJ, 684, 1240

Favre C. et al., 2018, ApJ, 859, 136

Fedoseev G., Ioppolo S., Zhao D., Lamberts T., Linnartz H., 2015, MNRAS, 446, 439

Fedoseev G., Chuang K. J., Ioppolo S., Qasim D., van Dishoeck E. F., Linnartz H., 2017, ApJ, 842, 52

Fontani F. et al., 2017, A\&A, 605, A57

Fuchs G. W., Cuppen H. M., Ioppolo S., Romanzin C., Bisschop S. E., Andersson S., van Dishoeck E. F., Linnartz H., 2009, A\&A, 505, 629

Gaches B. A. L., Offner S. S. R., Bisbas T. G., 2019, ApJ, 878, 105

Garrod R. T., 2013, ApJ, 765, 60

Garrod R. T., Pauly T., 2011, ApJ, 735, 15

Gaudel M. et al., 2020, A\&A, 637, A92

Gieser C. et al., 2019, A\&A, 631, A142

Goodman A. A., Benson P. J., Fuller G. A., Myers P. C., 1993, ApJ, 406, 528

Guzmán A. E., Guzmán V. V., Garay G., Bronfman L., Hechenleitner F., 2018, ApJS, 236, 45

Harsono D., Visser R., Bruderer S., van Dishoeck E. F., Kristensen L. E., 2013, A\&A, 555, A45

Hasegawa T. I., Herbst E., 1993, MNRAS, 261, 83

Hasegawa T. I., Herbst E., Leung C. M., 1992, ApJS, 82, 167

Herbst E., van Dishoeck E. F., 2009, ARA\&A, 47, 427

Hincelin U., Commerçon B., Wakelam V., Hersant F., Guilloteau S., Herbst E., 2016, ApJ, 822, 12

Hosokawa T., Omukai K., 2009, ApJ, 691, 823

Ioppolo S., Cuppen H. M., Linnartz H., 2011, Rendiconti Lincei, 22, 211

Jacobsen S. K. et al., 2018, A\&A, 612, A72

Jessop N. E., Ward-Thompson D., 2000, MNRAS, 311, 63

Jørgensen J. K. et al., 2016, A\&A, 595, A117

Jørgensen J. K. et al., 2018, A\&A, 620, A170

Karnath N. et al., 2020, ApJ, 890, 129

Keto E., Caselli P., 2008, ApJ, 683, 238

Lada C. J., 1987, in Peimbert M., Jugaku J., eds, IAU Symp.Vol. 115, Star Forming Regions. Cambridge University Press, Cambridge, UK, p. 1

Lee J.-E. et al., 2019, Nature Astron., 3, 314

Lee C. W., Myers P. C., Tafalla M., 1999, ApJ, 526, 788

Leger A., Jura M., Omont A., 1985, A\&A, 144, 147

Leurini S. et al., 2016, A\&A, 595, L4

Li X., Heays A. N., Visser R., Ubachs W., Lewis B. R., Gibson S. T., van Dishoeck E. F., 2013, A\&A, 555, A14

Li Z. Y., Banerjee R., Pudritz R. E., Jørgensen J. K., Shang H., Krasnopolsky R., Maury A., 2014, in Beuther H., Klessen R. S., Dullemond C. P., Henning T., eds, Protostars and Planets VI. The University of Arizona Press, Tucson, AZ, USA, p. 173

Ligterink N. F. W. et al., 2017, MNRAS, 469, 2219

Manigand S. et al., 2019, A\&A, 623, A69

Manigand S. et al., 2020, preprint (arXiv:2007.04000)

Maret S. et al., 2020, A\&A, 635, A15

Maret S., Belloche A., Maury A. J., Gueth F., André P., Cabrit S., Codella C., Bontemps S., 2014, A\&A, 563, L1

Masunaga H., Inutsuka S.-i., 2000, ApJ, 531, 350

Maureira M. J., Pineda J. E., Segura-Cox D. M., Caselli P., Testi L., Lodato G., Loinard L., Hernandez-Gomez A., 2020, ApJ, 897, 59

McElroy D., Walsh C., Markwick A. J., Cordiner M. A., Smith K., Millar T. J., 2013, A\&A, 550, A36

McGuire B. A., 2018, ApJS, 239, 17

Noble J. A., Dulieu F., Congiu E., Fraser H. J., 2011, ApJ, 735, 121

Öberg K. I., van Dishoeck E. F., Linnartz H., 2009a, A\&A, 496, 281

Öberg K. I., Garrod R. T., van Dishoeck E. F., Linnartz H., 2009b, A\&A, 504, 891

Öberg K. I., Linnartz H., Visser R., van Dishoeck E. F., 2009c, ApJ, 693, 1209

Padovani M., Marcowith A., Hennebelle P., Ferrière K., 2016, A\&A, 590, A8 
Padovani M., Galli D., Ivlev A. V., Caselli P., Ferrara A., 2018, A\&A, 619, A144

Palla F., Stahler S. W., 1991, ApJ, 375, 288

Penteado E. M., Walsh C., Cuppen H. M., 2017, ApJ, 844, 71

Podio L., Lefloch B., Ceccarelli C., Codella C., Bachiller R., 2014, A\&A, 565, A64

Robitaille T. P., Whitney B. A., Indebetouw R., Wood K., Denzmore P., 2006, ApJS, 167, 256

Ruaud M., Wakelam V., Hersant F., 2016, MNRAS, 459, 3756

Schöier F. L., Jørgensen J. K., van Dishoeck E. F., Blake G. A., 2002, A\&A, 390,1001

Schwarz K. R., Bergin E. A., Cleeves L. I., Zhang K., Öberg K. I., Blake G. A., Anderson D., 2018, ApJ, 856, 85

Shingledecker C. N., Tennis J., Le Gal R., Herbst E., 2018, ApJ, 861, 20

Shingledecker C. N., Vasyunin A., Herbst E., Caselli P., 2019, ApJ, 876, 140

Shu F. H., 1977, ApJ, 214, 488

Shu F. H., Adams F. C., Lizano S., 1987, ARA\&A, 25, 23

Sipilä O., Caselli P., Taquet V., 2016, A\&A, 591, A9

Swings P., Rosenfeld L., 1937, ApJ, 86, 483

Taquet V., Charnley S. B., Sipilä O., 2014, ApJ, 791, 1

Tielens A. G. G. M., Hagen W., 1982, A\&A, 114, 245

Vasyunin A. I., Caselli P., Dulieu F., Jiménez-Serra I., 2017, ApJ, 842, 33

Visser R., Dullemond C. P., 2010, A\&A, 519, A28

Visser R., van Dishoeck E. F., Doty S. D., Dullemond C. P., 2009, A\&A, 495, 881

Visser R., Doty S. D., van Dishoeck E. F., 2011, A\&A, 534, A132

Wakelam V. et al., 2017, Mol. Astrophys., 9, 1

Wakelam V., Vastel C., Aikawa Y., Coutens A., Bottinelli S., Caux E., 2014, MNRAS, 445, 2854

Wakelam V., Chapillon E., Dutrey A., Guilloteau S., Iqbal W., Coutens A., Majumdar L., 2019, MNRAS, 484, 1563

Walsh C., Millar T. J., Nomura H., Herbst E., Widicus Weaver S., Aikawa Y., Laas J. C., Vasyunin A. I., 2014, A\&A, 563, A33

Watanabe N., Kouchi A., 2008, Progr. Surf. Sci., 83, 439

Young C. H., Evans Neal J. I., 2005, ApJ, 627, 293

Zhao B., Caselli P., Li Z.-Y., 2018, MNRAS, 478, 2723

\section{APPENDIX A: INITIAL ABUNDANCES}

This section provides additional information about the abundances before and after the prestellar phase. In Table A1, the atomic abundances relative to $\mathrm{n}_{\mathrm{H}}$ at the beginning of the precollapse phase
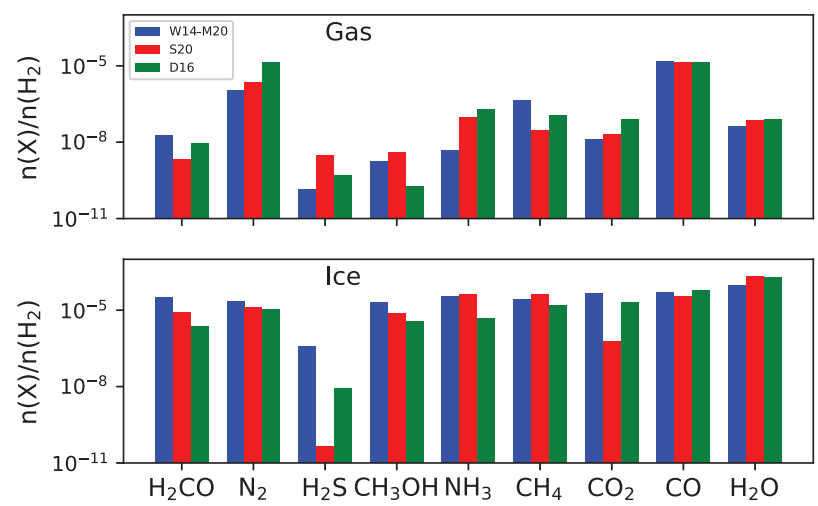

Figure A1. Molecular precollapse abundances (Table A2) at the start of the collapse phase relative to $\mathrm{n}_{\mathrm{H}_{2}}$. Blue bars describe the values of the W14-M20 model, red bars describe the S20 model, and green bars describe the D16 model. The upper panel depicts the gases, the lower panel shows the ices. For the three-phase chemical models (NAUTILUS and MAGICKAL, used in W14-M20 and S20, respectively), the bulk and surface have been summed to obtain the total abundances in the ice.
Table A1. Initial atomic abundances at the start of the precollapse phase of the three studied models relative to $\mathrm{n}_{\mathrm{H}}$.

\begin{tabular}{lccc}
\hline & ${\mathrm{W} 14-M 20^{a}}^{a}$ & $\mathrm{~S}^{2} 0^{b}$ & $\mathrm{D}^{\circ} 6^{c}$ \\
\hline $\mathrm{H}$ & - & $1.0 \times 10^{-4}$ & $5.0 \times 10^{-5}$ \\
$\mathrm{H}_{2}$ & 0.5 & 0.49995 & 0.5 \\
$\mathrm{He}$ & $9.0 \times 10^{-2}$ & $9.0 \times 10^{-2}$ & $9.8 \times 10^{-2}$ \\
$\mathrm{C}^{\mathrm{d}}$ & $1.7 \times 10^{-4}$ & $1.4 \times 10^{-4}$ & $1.4 \times 10^{-4}$ \\
$\mathrm{~N}$ & $6.2 \times 10^{-5}$ & $7.0 \times 10^{-5}$ & $7.5 \times 10^{-5}$ \\
$\mathrm{O}$ & $2.4 \times 10-4$ & $3.2 \times 10^{-4}$ & $3.2 \times 10^{-4}$ \\
$\mathrm{Na}$ & - & $2.0 \times 10^{-8}$ & $2.0 \times 10^{-9}$ \\
$\mathrm{Mg}$ & - & $7.0 \times 10^{-9}$ & $7.0 \times 10^{-9}$ \\
$\mathrm{Si}$ & - & $8.0 \times 10^{-9}$ & $8.0 \times 10^{-9}$ \\
$\mathrm{P}$ & - & $3.0 \times 10^{-9}$ & $3.0 \times 10^{-9}$ \\
$\mathrm{~S}^{\mathrm{d}}$ & $1.5 \times 10^{-6}$ & $8.0 \times 10^{-8}$ & $8.0 \times 10^{8}$ \\
$\mathrm{Cl}^{\mathrm{d}}$ & $1.0 \times 10^{-9}$ & $4.0 \times 10^{-9}$ & $4.0 \times 10^{-9}$ \\
$\mathrm{Fe}^{\mathrm{d}}$ & $1.0 \times 10^{-8}$ & $3.0 \times 10^{-9}$ & $3.0 \times 10^{-9}$ \\
$\mathrm{~F}$ & $6.68 \times 10^{-9}$ & - & - \\
\hline
\end{tabular}

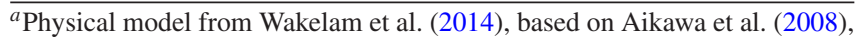
last published in Manigand et al. (2020); ${ }^{b}$ Stéphan et al. (in preparation); ${ }^{c}$ last published in Drozdovskaya et al. (2016); ${ }^{d}$ in the case of W14-M20 these elements are fully in their ionic form $\left(\mathrm{C}^{+}, \mathrm{S}^{+}, \mathrm{Cl}^{+}\right.$, and $\left.\mathrm{Fe}^{+}\right)$.

Table A2. Precollapse molecular abundances for the three studied models relative to $\mathrm{n}_{\mathrm{H}_{2}}$ at the start of the collapse phase.

\begin{tabular}{lccc}
\hline & $\mathrm{W} 14-\mathrm{M} 20^{a}$ & $\mathrm{~S} 20^{b}$ & $\mathrm{D} 16^{c}$ \\
\hline $\mathrm{Gas}$ & & & \\
$\mathrm{H}_{2} \mathrm{CO}$ & $1.85 \times 10^{-8}$ & $2.09 \times 10^{-9}$ & $7.73 \times 10^{-8}$ \\
$\mathrm{~N}_{2}$ & $1.10 \times 10^{-6}$ & $2.38 \times 10^{-6}$ & $1.35 \times 10^{-5}$ \\
$\mathrm{H}_{2} \mathrm{~S}$ & $1.39 \times 10^{-10}$ & $3.01 \times 10^{-9}$ & $5.28 \times 10^{-10}$ \\
$\mathrm{CH}_{3} \mathrm{OH}$ & $1.77 \times 10^{-9}$ & $4.26 \times 10^{-9}$ & $1.97 \times 10^{-10}$ \\
$\mathrm{NH}_{3}$ & $4.71 \times 10^{-9}$ & $9.52 \times 10^{-8}$ & $2.08 \times 10^{-7}$ \\
$\mathrm{CH}_{4}$ & $4.56 \times 10^{-7}$ & $2.86 \times 10^{-8}$ & $1.16 \times 10^{-7}$ \\
$\mathrm{CO}_{2}$ & $1.34 \times 10^{-8}$ & $2.00 \times 10^{-8}$ & $7.89 \times 10^{-8}$ \\
$\mathrm{CO}^{-8}$ & $1.56 \times 10^{-5}$ & $1.41 \times 10^{-5}$ & $1.39 \times 10^{-5}$ \\
$\mathrm{H}_{2} \mathrm{O}$ & $4.19 \times 10^{-8}$ & $7.58 \times 10^{-8}$ & $7.73 \times 10^{-8}$ \\
$\mathrm{Ice}$ & - & & - \\
$\mathrm{H}_{2} \mathrm{CO}$ & $3.07 \times 10^{-5}$ & $8.31 \times 10^{-6}$ & $2.32 \times 10^{-6}$ \\
$\mathrm{~N}_{2}$ & $2.26 \times 10^{-5}$ & $1.27 \times 10^{-5}$ & $1.09 \times 10^{-5}$ \\
$\mathrm{H}_{2} \mathrm{~S}$ & $3.93 \times 10^{-7}$ & $4.21 \times 10^{-11}$ & $8.11 \times 10^{-9}$ \\
$\mathrm{CH}_{3} \mathrm{OH}$ & $2.08 \times 10^{-5}$ & $7.80 \times 10^{-6}$ & $3.66 \times 10^{-6}$ \\
$\mathrm{NH}_{3}$ & $3.41 \times 10^{-5}$ & $4.25 \times 10^{-5}$ & $4.82 \times 10^{-6}$ \\
$\mathrm{CH}_{4}$ & $2.67 \times 10^{-5}$ & $3.97 \times 10^{-5}$ & $1.54 \times 10^{-5}$ \\
$\mathrm{CO}_{2}$ & $4.53 \times 10^{-5}$ & $5.86 \times 10^{-7}$ & $5.92 \times 10^{-5}$ \\
$\mathrm{CO}^{-5}$ & $4.85 \times 10^{-5}$ & $3.32 \times 10^{-5}$ & $1.39 \times 10^{-5}$ \\
$\mathrm{H}_{2} \mathrm{O}$ & $9.53 \times 10^{-5}$ & $2.12 \times 10^{-4}$ & $1.87 \times 10^{-4}$ \\
\hline
\end{tabular}

${ }^{a}$ Physical model from Wakelam et al. (2014), based on Aikawa et al. (2008), last published in Manigand et al. (2020); ${ }^{\mathrm{b}}$ Stéphan et al. (in preparation); ${ }^{\mathrm{c}}$ last published in Drozdovskaya et al. (2016).

are presented. These are used to compute the initial molecular abundances that are used as input for the chemical modelling of the collapse. These abundances are given in Table A2 and are plotted for the gas and the ice in Fig. A1.

\section{APPENDIX B: INDIVIDUAL ABUNDANCES}

Here, the evolution of the abundances of the individual molecular species in the gas and in the ice are presented. Furthermore, an updated version of Fig. 6 with results obtained with the two-phase chemical network is depicted. 


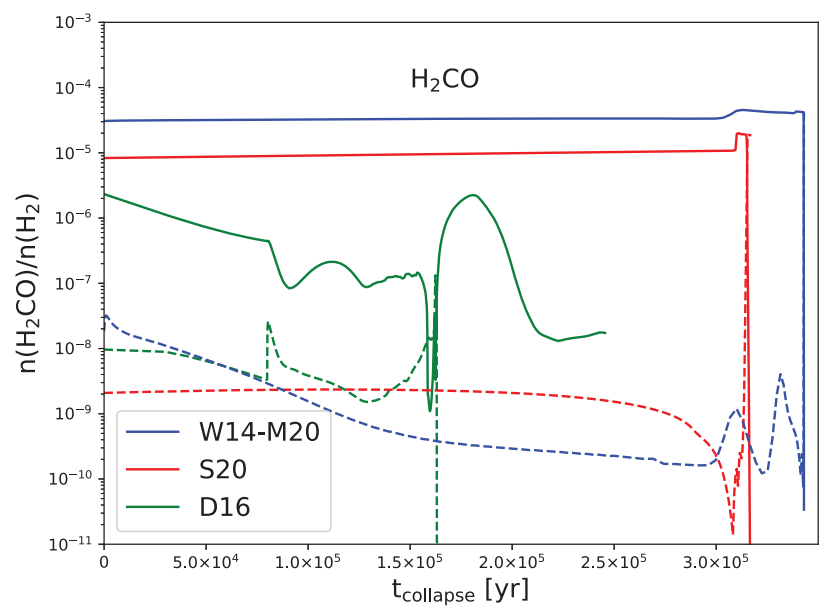

Figure B1. This figure shows the evolution of $\mathrm{H}_{2} \mathrm{CO}$ throughout the collapse. The collapse time in years is plotted on the $x$-axis, the abundance relative to $\mathrm{n}_{\mathrm{H}_{2}}$ is plotted on the $y$-axis. Dashed lines represent $\mathrm{H}_{2} \mathrm{CO}_{\text {gas }}$, solid lines $\mathrm{H}_{2} \mathrm{CO}_{\text {ice }}$. The trajectory of 62.4 au from the W14-M20 model is depicted in blue, the 49.7 au from S20, in red and the 46.7 au from D16 in green. For the three-phase chemical models (NAUTILUS and MAGICKAL, used in W14-M20 and S20, respectively), the bulk and surface have been summed to obtain the total abundances in the ice. See Figs B1-B10.

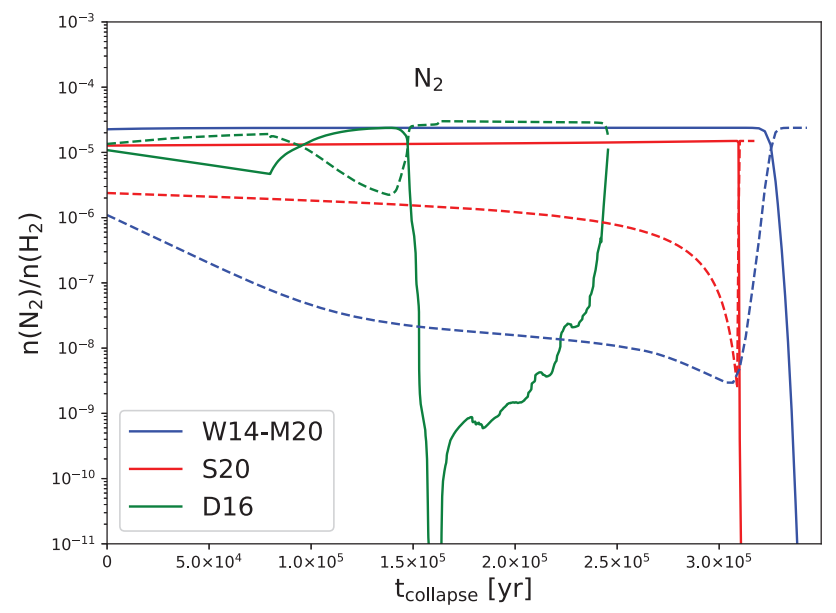

Figure B2. Same as Fig. B1, but for $\mathrm{N}_{2}$.

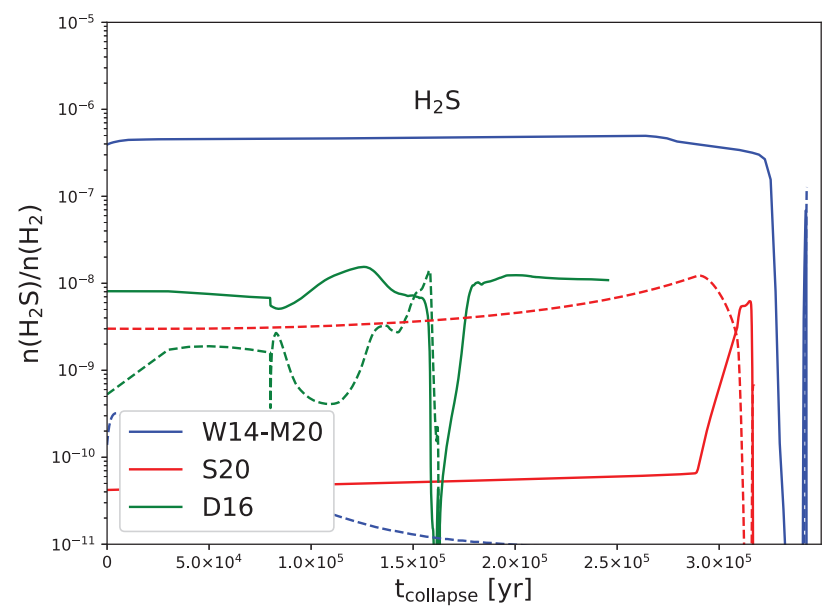

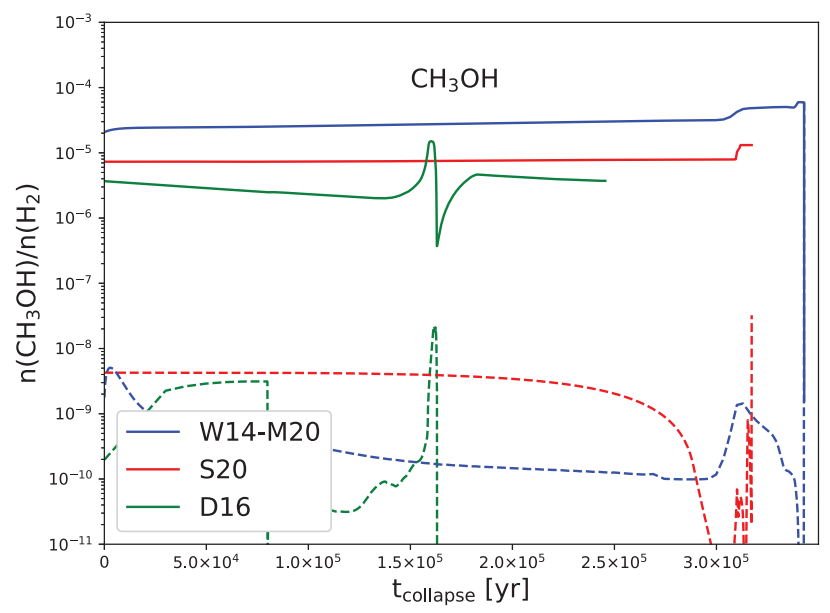

Figure B4. Same as Fig. B1, but for $\mathrm{CH}_{3} \mathrm{OH}$.

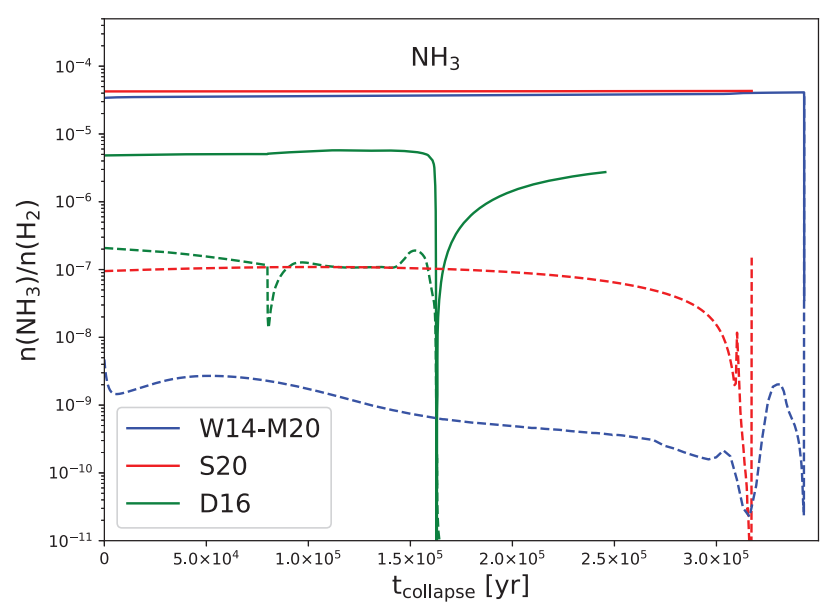

Figure B5. Same as Fig. B1, but for $\mathrm{NH}_{3}$.

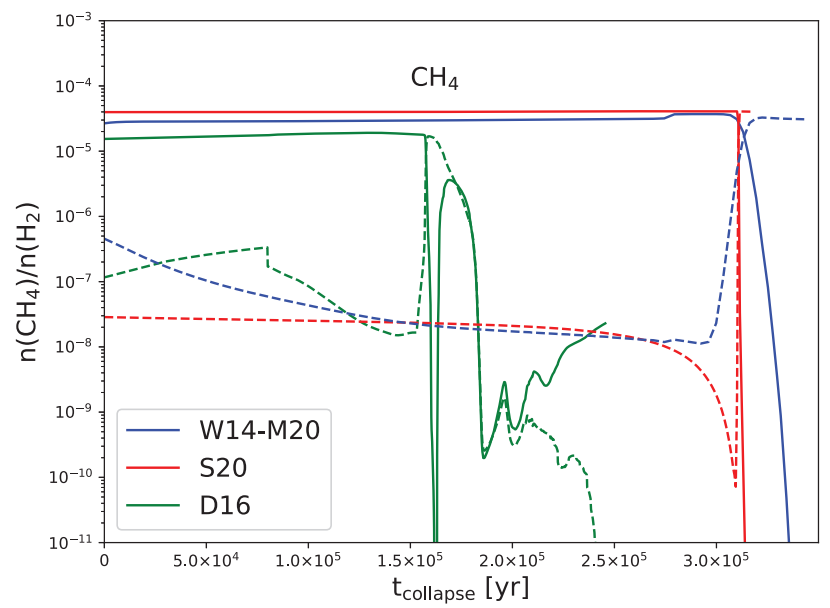

Figure B6. Same as Fig. B1, but for $\mathrm{CH}_{4}$.

Figure B3. Same as Fig. B1, but for $\mathrm{H}_{2} \mathrm{~S}$. 


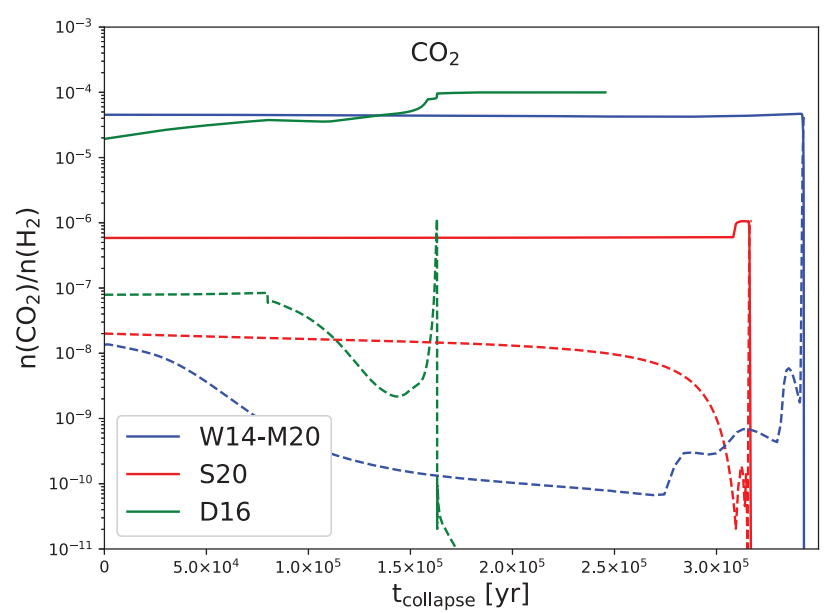

Figure B7. Same as Fig. B1, but for $\mathrm{CO}_{2}$.

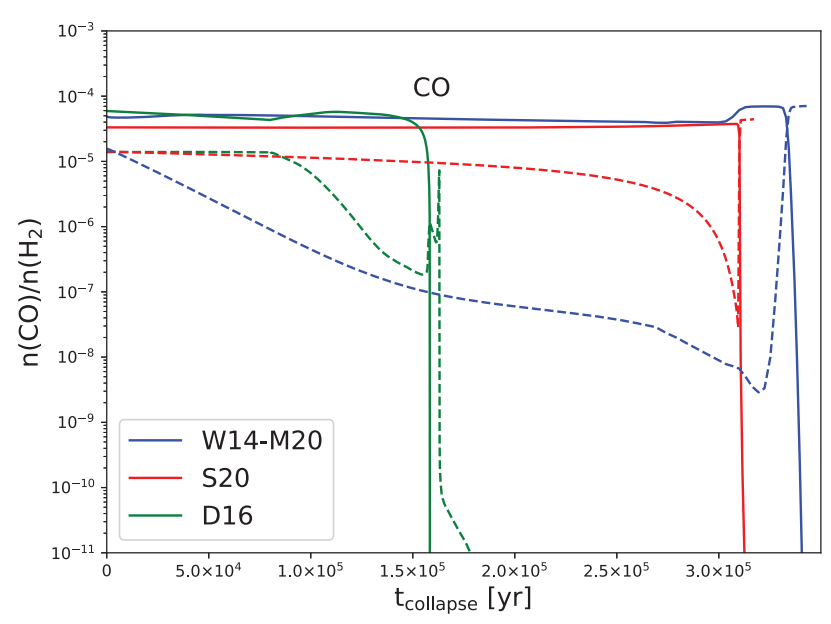

Figure B8. Same as Fig. B1, but for CO.

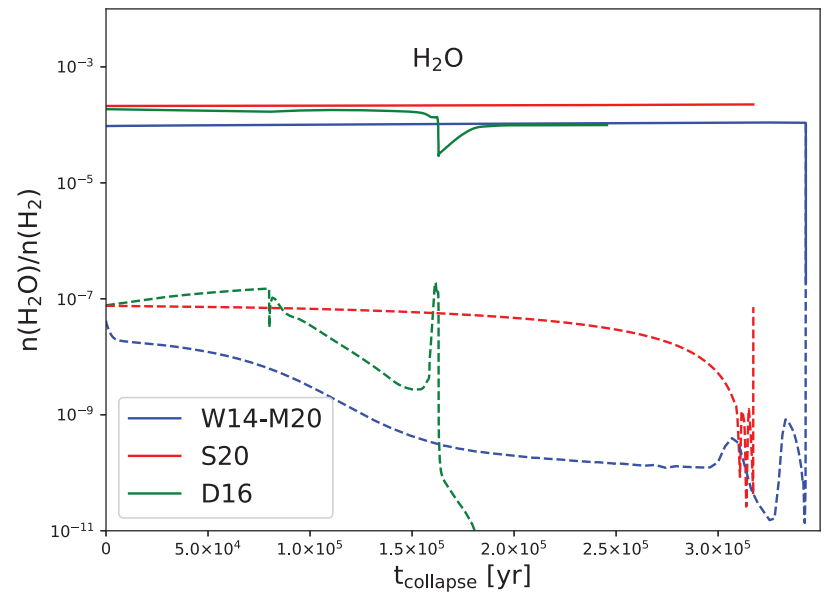

Figure B9. Same as Fig. B1, but for $\mathrm{H}_{2} \mathrm{O}$.

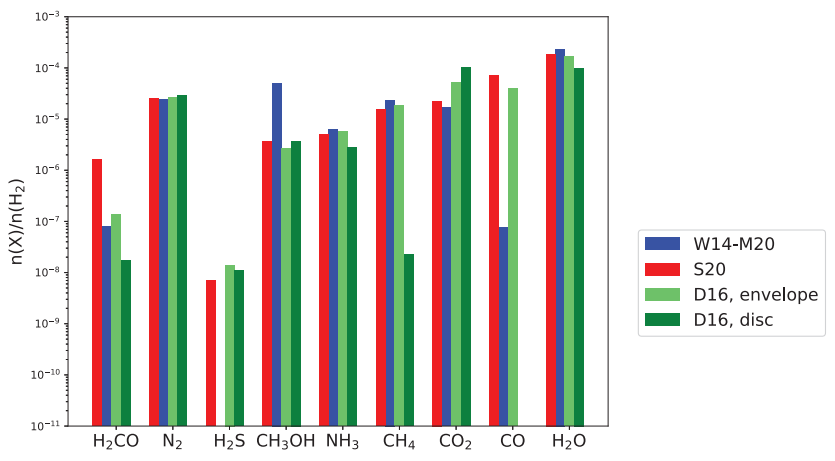

Figure B10. Postcollapse molecular abundances relative to $\mathrm{n}_{\mathrm{H}_{2}}$ with the two-phase D16 chemical model. Blue bars describe the values of the W14M20 model (final position at $62.4 \mathrm{au}$ ), red bars show the S20 model (final position at $49.7 \mathrm{au}$ ), light green bars correspond to the abundances in the warm envelope upon disc entry in the D16 model (at $t=1.5 \times 10^{5} \mathrm{yr}$ $=0.61\left(t_{\text {collapse }}^{\mathrm{D} 16}\right)$, dark green bars illustrate the abundances in the disc at the end of the collapse in the D16 model (final position at $46.7 \mathrm{au}$ ).

This paper has been typeset from a $\mathrm{T}_{\mathrm{E}} \mathrm{X} / \mathrm{L} \mathrm{T} \mathrm{E} \mathrm{X}$ file prepared by the author. 